\title{
HIERARCHICAL NONLINEAR DYNAMICS OF HUMAN ATTENTION
}

\author{
Mikhail Rabinovich ${ }^{1}$, Irma Tristan ${ }^{1}$ and Pablo Varona ${ }^{2 *}$ \\ ${ }^{1}$ BioCircuits Institute. University of California, San Diego. 9500 Gilman Drive \#0328. La \\ Jolla, CA 92093-0328. \\ ${ }^{2}$ Grupo de Neurocomputación Biológica, Dpto. de Ingeniería Informática. Escuela \\ Politécnica Superior. Universidad Autónoma de Madrid. 28049 Madrid. Spain. \\ *Corresponding author: pablo.varona@uam.es
}

\begin{abstract}
Attention is the process of focusing mental resources on a specific cognitive/behavioral task. Such brain dynamics involves different partially overlapping brain functional networks whose interconnections change in time according to the performance stage, and can be stimulus-driven or induced by an intrinsically generated goal. The corresponding activity can be described by different families of spatiotemporal discrete patterns or sequential dynamic modes. Since mental resources are finite, attention modalities compete with each other at all levels of the hierarchy, from perception to decision making and behavior. Cognitive activity is a dynamical process and attention possesses some universal dynamical characteristics. Thus, it is time to apply nonlinear dynamical theory for the description and prediction of hierarchical attentional tasks. Such theory has to include the analyses of attentional control stability, the time cost of attention switching, the finite capacity of informational resources in the brain, and the normal and pathological bifurcations of attention sequential dynamics. In this paper we have integrated today's knowledge, models and results in these directions.
\end{abstract}

Keywords: Attention sharing; perception binding; stable heteroclinic channel; transient dynamics; robust sequence generation; working memory capacity; temporal coordination and creativity. 


\section{Contents}

1. Introduction. Attention as a dynamical process

1.1 Functional networks of attention

1.2. Attention and mind

2. Transients. Low-dimensional cognitive dynamics

2.1. Projection functions. Global cognitive modes. Canonic model

2.2. Metastable states. Stable heteroclinic channels

3. Stability and coordination dynamics of the sequences

3.1. Finite information processing resources Attention and working memory capacity - stability limit

3.2. Intrinsic attentional dynamics - sequence generation and the perception of the arrow of time

3.3. Brain-to-brain temporal coordination

4. Dynamics of attention sharing, switching and decision making

4.1. Low-dimensional mind dynamics

4.2. Attention sharing and perception binding

4.3, Information flows in the phase space

4.4. Decision making strategy

4.5. Attention -pain interaction

5. Attentional hierarchy: creativity and disorders

5.1. Attention - emotion joint dynamics. Mental disorders

5.2. Creativity: Attentional control turn off?

5.3. Attention dynamics and creative thinking. Divergence-convergence bifurcation

6. Conclusions

7. Appendices

7.1 Robustness of sequential cognitive dynamics based on heteroclinic sequences

7.2. Simulation parameters

8. References 


\section{Introduction. Attention as a dynamical process}

One of the most challenging questions in Nature is 'How does the activity of a vast number of brain elements - neurons, synapses, glia cells, etc. - transform into the dynamics of cognitive functions in the human mind?' The modeling of cognitive and, in particular, attention dynamics is not an easy subject for neuro- and cognitive sciences. Experiments addressing this topic with state of the art techniques such as functional magnetic resonance imaging (fMRI), near infrared spectroscopy (NIRS), electroencephalography (EEG), etc. are challenging and to some extent limited and hard to interpret. The same applies to behavioral experiments when analyzing their relation to physiological recordings. Thus, attention dynamics must be addressed with theoretical approaches that rely on incomplete experimental data. A few authors have proposed models of attention dynamics that describe individual neurons and network activity combined with theoretical principles such as biased competition (Desimone and Duncan, 1995; Dieter and Tadin, 2011) and hierarchical network organization, e.g. see (Deco and Rolls, 2005, 2003). Several of these models use attractor dynamics as the main computational ingredient. In this paper, we argue that cognitive neuroscience in general, and attention studies in particular, need to bridge the gap between the large amount of experimental efforts and the theoretical approaches by relying on perspectives that go beyond specific bottom-up, or exclusively top-down reductionist paradigms, see also (Deco et al., 2008). To achieve this goal, we look into dynamical principles that can describe robust transient and hierarchical sequential neural activity at multiple temporal and spatial scales, characterize the interaction between limited mental resources and represent complex and history-dependent intrinsic dynamics and external stimuli. This approach can result in predictive behavioral models independently of their bottom-up or top-down design. Furthermore, this strategy is not only important to provide a computational perspective on cognitive functions (Cowell et al., 2012; Meehan and Bressler, 2012; Park and Friston, 2013), but also to understand several diseases that may have a dynamical origin and thus could be better explained and treated using dynamical insights. The new theoretical paradigms that arise from the combination of experimental analysis and top-down and bottom-up models will lead to novel techniques and experiments in the pursuit of unveiling attention hierarchical dynamics (see Figure 1). 


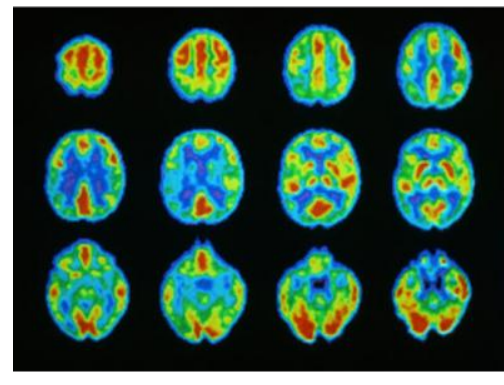

Brain imaging

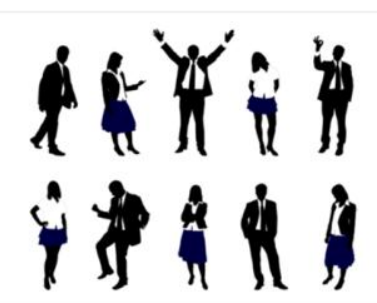

Behavior, attention diseases, psychophysiology

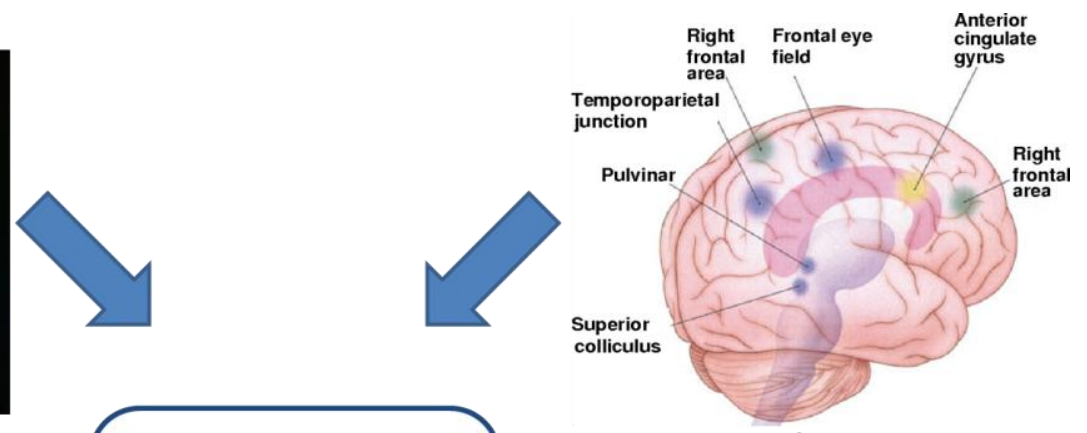

Anatomy of attention networks

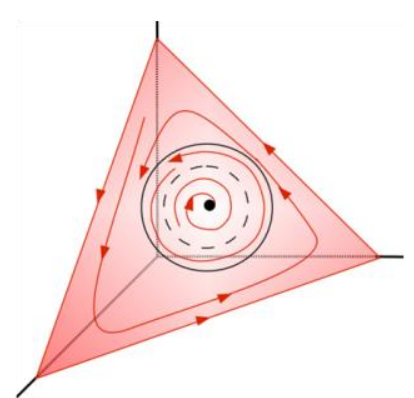

Theoretical approaches on robust transient dynamics

Figure 1 (Color online). Integrative approach to the modeling of attention dynamics. New dynamical paradigms of attention arise from the combination of experimental analysis, including behavior and pathological studies, and top-down and bottom-up models that use anatomical and physiological knowledge to unveil attention hierarchical dynamics.

Modeling of a cognitive process requires: (i) knowledge about the spatial structure of the specialized brain networks (and their dynamical interconnections) that participate in the performance of the cognitive process (Hermundstad et al., 2013; Park and Friston, 2013), and (ii) the formulation of the main dynamical principles that control this processing. Such knowledge makes possible to reconstruct the projection functions that connect the anatomical space with the parameters of dynamical model (the functional space). In principle, these functions can be estimated from brain imaging experiments (Makeig et al., 2009; Pereira et al., 2009; Viviani et al., 2005; Wen-Kai Tsai et al., 2012) and the dynamical principles can be formulated based on the observed brain activity, behavioral experiments and intuition derived from human every-day experience. Let us discuss these principles.

In last decade neurophysiological and imaging experiments have shown that the human brain never rests - it demonstrates modulated transient activity even at "rest" 
(Deco et al., 2013; Raichle, 2011; Segall et al., 2012). Nevertheless, in spite that cognitive processes are transient, we think, speak and generate behavioral programs in a very robust way. We name robust transientness as the first brain dynamical principle.

Robust brain activity is at the same time extremely sensitive to informational signals from intrinsic or external sources. These signals can be in the form of a visual sensory input that informs you about a danger on the road, or an emotional input that changes the strategy of your social behavior (George, 2000; Pessoa, 2010; Turner and Stets, 2005). Thus, the second principle is - the sensitivity of brain activity to informational inputs. Evidently, the first two principles partially contradict each other.

A third brain dynamical principle is related to mental resources: brain information processing resources are limited (Marois and Ivanoff, 2005; Slagter et al., 2007) everyone has worried at some point about the very low capacity of their working memory and about the problem of simultaneously keeping attention on more than two or three different subjects or activities. This principle also raises many questions because of the existing contradiction with the fact that the brain is able to perform what it seems a countless number of different cognitive tasks. Below we will show how the dynamical approach is able to help solving the contradictions mentioned above.

Of course, the choice of the key dynamical principles to model attention is not unique. In his paper we emphasize those that can describe robust transient and hierarchical sequential activity, but there are other phenomena to consider. For example noise and time delay can play a critical role in expanding the dynamical repertoire in cognitive dynamics (Deco et al., 2011, 2009). Here we concentrate on describing a dynamical model which is simple as possible by choosing just a few of key dynamical principles that provide robustness and at the same time sensitivity to incoming information.

\subsection{Functional networks of attention.}

Attention, as other cognitive functions in humans, is a multidimensional and episodic process (Duncan, 2013). Attention, in particular, includes the ability to focus and select what is important at a given time and space, the aptitude to sustain mental efforts while performing tasks that require mental resources and to inhibit thoughts and actions while considering alternatives. Thus, attention is a process that includes remaining alert and focused, selecting what we should attend to, overlook what we should not, and sustaining this focusing for the right duration. Attention allows us to sequentially plan our thoughts and actions and, in fact, participate in the prediction of the future: "Attention is expectation, and there is no consciousness without a certain attention to life" (Bergson, 1920). Attention is often seen as a virtual spotlight through which the brain processes information about the external and internal worlds. 
There are two major forms of attention: passive and active (Gaddes and Edgell, 1994). Passive attention is an involuntary process driven by external events from the environment, such as a sudden auditory, visual or olfactory stimulus. Passive attention does not involve a conscious effort. On the other hand, active attention is voluntary and is driven by interest, concentration and vigilance (Posner and Boies, 1971), thus it needs abilities such as curiosity and gumption, and also involves conscious effort.

On the basis of detailed neuroanatomical, electrophysiological and neurochemical studies in animals, as well as human neuroimaging data, researchers (see (Bressler and Menon, 2010; Goldman-Rakic, 1988; Mesulam, 1998)) have identified large-scale cortical-subcortical circuits, including feedback loops, which perform different functional attentional and working memory processes (Posner et al., 2006; Rissman and Wagner, 2012). The attentional "reorienting" from one subject - modality to another involves the coordinated dynamics of a right hemisphere network that interrupts and resets ongoing activity, and of another network specialized for selecting and linking stimuli to responses. During resting states, each network is distinct and internally correlated, but when attention is focused, the first network is suppressed to avoid distracting events. The different recruitment processes may reflect inputs to the first attention network from systems involved in controlling the switching dynamics between networks (Corbetta et al., 2008).

At any given moment, multiple thoughts or representations compete for limited attentional resources and for the control of sequential behavior. Researchers agree that attention involves the existence of top-down signals that play a key role in resolving this competition by selectively enhancing the modalities that underlie perceptions, while inhibiting irrelevant subjects (e.g., (Mao and Wang, 2008)). Because inhibition is crucially important for cognitive dynamics, we need to describe these processes in more detail.

The concepts of inhibition and interference control date back over 100 years (for reviews, see (Dempster and Brainerd, 1995; Macleod et al., 2003)). Cognitiveprocessing studies also early faced the problem of the limitation of cognitive resources (e.g., memory capacity and processing efficiency) to explain attention (Kahneman, 1973; Norman and Bobrow, 1975). Inhibition and interference control have been studied from different viewpoints in neuroscience research (Dagenbach and Carr, 1994; Dempster and Brainerd, 1995; Sarason et al., 1996). For example, deficient inhibition-related processes have been described in disorders such as attentiondeficit/hyperactivity disorder (Barkley, 1997; Nigg, 2001), schizophrenia (Nestor et al., 2010), autism (Ciesielski and Harris, 1997), and obsessive-compulsive disorder (Clayton et al., 1999). We will discuss this in the last section of the paper. A popular point of view supposes that inhibition-related processes are a family of different mechanisms rather than a single unitary construct. For a critical review of this topic see (Ben-Simon et al., 2013; Young et al., 2009). 
Large-scale functional brain networks usually operate on the edge of instability (Deco and Jirsa, 2012; Ghosh et al., 2008). In this state, the networks are in a low firing stable state and they are ready for several possible configurations. Small extrinsic or intrinsic stimuli are able to form and shape the cognitive task-related networks. The resting state reflects the dynamical capabilities of the brain, which emphasizes the crucial interplay of time and space (Deco et al., 2013). Hasenkamp et al. have studied how attentional and resting (default mode) networks work together (Hasenkamp et al., 2012). Their results suggest that the default mode network is active during mind wandering, which is often experienced intermittently during sustained attention tasks. Conversely, an anticorrelated task-positive network is thought to participate in various forms of attentional processing.

\subsection{Attention and mind.}

Attention plays a key role in linking brain and mind. We already mentioned the attentional networks that project brain activities to cognitive functions. Let us consider the feedback from mind to brain through attentional control, learning and memory. This includes learning, short term memory -working memory, decision making and other cognitive processes that functionally change brain networks under the attentional supervision. In fact, attention is related to all mental activities including consciousness.

Attention and consciousness are responsible for the low-dimensionality of mind dynamics. Rephrasing William James, one can say: both attention and consciousness are hierarchical dynamical processes. During such processes a short-term integration coherentization- can be implemented by differentiated brain metastable states through the continuous interplay between the environment, the body, and the brain itself. The dynamic structure underlying successive metastable states along such transient processes is a key point for understanding the relationship between attention and consciousness. Because both attention and consciousness choose specific transients (e.g. as chains of metastable states in the functional space of the mind - see 2.2.), the dimension of mental processes under the control of attention or consciousness has to be much lower compared with uncontrollable processes. This view is supported by results of imaging analyses. In particular, in (Lee et al., 2009), authors have shown that a conscious process displayed a lower dimensional dynamics that changed to a randomlike process after loss of consciousness.

Even though the brain networks responsible for attention and consciousness strongly overlap, already at the neural level, one can see dissociation between these two processes. Because attention and consciousness clearly interact at the cognitive and behavioral levels, there is a strong debate about their independence (see, for example, (Koch and Tsuchiya, 2007; Lamme, 2003)). In our point of view, one can build an integrated dynamical model as the one that we will present for the description of the 
attention and perception (see section 4) in which attention and consciousness correspond to distinct neural mechanisms feeding a single decision process leading to behavior. In this view, consciousness should not be considered as a top-level executive function. Such approach is a reasonable perspective because both attention and consciousness are responsible for the observed stability of thinking and behavior in our daily life, and this is a key point that guarantees the low-dimensionality of the dynamics of the cognitive task performance.

Attention and working memory. - "The true art of memory is the art of attention", wrote Samuel Jonson (Johnson, 2012). Is this correct in the light of modern experiments? Multivariate pattern analyses of fMRI have shown that only items within the focus of attention induce an active neural trace in the brain (Lewis-Peacock et al., 2012). Activity corresponding to representations of items outside the attention focus quickly drop to baseline levels. However, as these authors showed, this information is remembered after a brief delay and the refocused attention to previously unattended information can restore the active neural signature of that information. Thus, it would be not completely right to say that these two functions coincide in spite that they are based on strongly overlapping brain networks (see also (Nobre and Stokes, 2011)).

We wish to remind here that attention is the set of functions which focus on information processing according to current cognitive task goals and expectations. This processing, in particular, may prepare the brain for foreseen events - their locations or their constituent features. It also involves selecting relevant items from a continuous stream of environmental information and inhibiting possible distractors. Short-term memory or working memory (Baddeley, 1992) keeps and represents the necessary items that are extracted, or built, from the stimulus and shapes the action. In spite that the chosen information (attention function) and the corresponding representations (working memory function) are different, from the dynamical point of view they are certainly two sides of the same phenomenon and often can be modeled together (Stedron et al., 2005).

Attention and learning of sequences. As we discussed above, attention actively selects and enhances information that is most relevant to the performance of a specific stage of a cognitive task or behavior. Learning enables perceptual and cognitive systems to use previous experience. The most intriguing question here is: How such networks are able to learn, keep and express information about sequential non-stationary cognitive or behavioral processes? To answer this question it is necessary: (i) to analyze the functional architecture of the brain networks responsible for the performance of specific sequential cognitive/behavioral activities (e.g. see (Wiestler and Diedrichsen, 2013)), (ii) to find the connection between them before and after learning and (iii) to 
find their mapping to mind/behavior, e.g. to find projection functions from brain to mind. Such program has not been completed yet as a whole. The first and second parts of the problem were partially solved, for example, in the work (Tamás Kincses et al., 2008).

From the modeling point of view, the learning of sequences can be seen as the formation, under sensory input and attentional control, of the topology of heteroclinic sequences in the phase space (we discuss this in detail in section 2.2, see also (Seliger et al., 2003)). More specifically, this means the formation of the connection matrix between metastable states in order to remember the corresponding sequential switching associated with a specific cognitive process (see Figure 2). In the absence of the attentional focus, spontaneous neural activity has a complex spatiotemporal structure that can be considered as a random pulsation of many cognitive modes. Cognitive or external stimuli and attentional queues choose the specific sequence of modes, and in the learning process the corresponding connection matrix is fixed. Thus, the memory of the transient cognitive processes keeps different potential heteroclinic chains of metastable states instead of traditional attractors. The focusing of attention on a specific cognitive task is responsible for the excitation of the specific set of metastable states and thus helps to avoid the overlapping of different task performances and warrants the low-dimensionality of the cognitive processing.

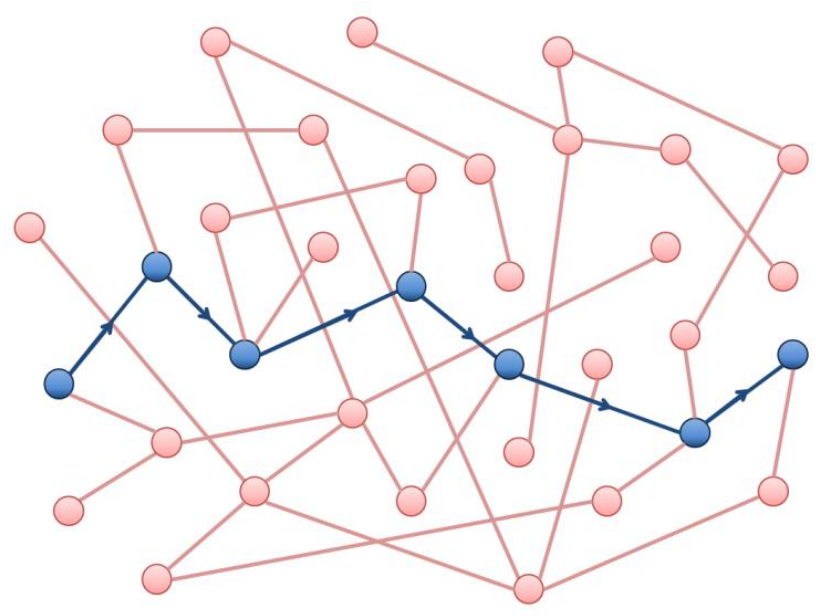

Figure 2 (Color online). Learned sequences: The figure illustrates the set of metastable states with potential (learned) connections. The static input supported by focused attention activates the subset of metastable states (depicted in blue) that form the heteroclinic channel corresponding to the specific transient output. The potential capacity of such sequential memory is easy to estimate (i.e. the number of permutations of all possible metastable states (Rabinovich et al., 2001)).

A similar approach, whose authors have named "memories-as-bifurcations", is discussed in (Kurikawa and Kaneko, 2013). The authors introduced an associative 
memory model with a sequential learning process. Memory recall in this model occurs when the spontaneous neural activity changes to an appropriate output activity upon application of an input, a bifurcation, wherein the input modifies the flow structure of the neural dynamics. In this model, learning is a process that helps create neural dynamical systems such that a target output is generated as an attractor upon a given input. Based on a simple Hebbian-type learning, the model is able to memorize a large number of input/output mappings. This dynamical view of memory processes complements and enhances the traditional viewpoint of "memories-as-attractors".

\section{Transients. Low-dimensional cognitive dynamics}

\subsection{Projection Functions. Global Cognitive Modes. Canonic model.}

To build a nonlinear dynamical theory of human attention dynamics, it is necessary to create a universal scale free model - a canonical model - of cognitive dynamical processes. We will do this based on the principles of brain dynamics formulated above. According to these principles the canonic model has to satisfy the following conditions: (i) equations have to be written for variables that can represent the evolution of brain elements (voxels) in their temporal coherency and have to have solutions that correspond to metastable patterns in the brain; (ii) the model is based on winnerless competitive dynamics -a nonlinear process of interaction of many agents that guarantees the sequential switching between metastable states and the robustness of transients (Rabinovich et al., 2001); (iii) the model is an open dissipative system where inhibition is balanced by excitation; and (iv) the model's dynamics has to be sensitive to the incoming information.

The reduction of high-dimensional brain data to a low-dimensional mind space is a very attractive idea that can be motivated by empirical observation. In particular, there are plenty of experiments that have illustrated the low-dimensionality of cognitive dynamics when it is governed by sensory stimuli. Formally, this means that large amounts of data can be represented by the dynamics of a reduced number of spatiotemporal patterns - modes - using spatiotemporal decomposition techniques, such as principal component analysis (Friston et al., 1993; Kelso et al., 1998; McIntosh et al., 1996; Pearson, 1901) and independent component analysis (Bell and Sejnowski, 1995; Makeig et al., 1997), for a review see (Banerjee et al., 2012).

To formalize our approach we name the spatial components of the modes as projection functions. These functions can be interpreted as signatures of large-scale networks that constitute the substrate on which the corresponding cognitive 
processing is based. Let us, first, formally introduce the cognitive modes and the projection functions. One can represent a cognitive process, like attention or working memory, in the form of linear combination of the modes

$$
R(l, t)=\sum_{m=1}^{M} P^{m}(l, t)
$$

where $P^{m}(l, t)=R^{m}(t) Q^{m}(l)$ is the $m$-th mode that depends on time and is based on the set of discrete coordinates $l$ in the brain space (voxels coordinates), $Q^{m}(l)$ is correspondingly the projection function of the $m$-th mode, and $M$ is the number of modes. We suppose that each mode satisfies a system of ordinary differential equation with the simplest square nonlinearity:

$$
\frac{d P^{m}}{d t}=P^{m}(l, t) \cdot\left[\tilde{\gamma}^{m}-\sum_{k=1}^{M} \tilde{\varsigma}^{m k} P^{k}(l, t)\right],
$$

Here $\tilde{\gamma}^{m}$ is the level of excitation, $\tilde{\varsigma}^{m k}$ is the level of inhibition of mode $m$ by mode $k$. After, substitution (2.1) and summation these equations take the form of the generalized Lotka-Volterra (GLV) model - canonic ecological model (Rabinovich et al., 2013)

$$
\theta_{m} \frac{d R^{m}}{d t}=R^{m} \cdot\left[\gamma^{m}\left(X^{m} S^{m}\right)-\sum_{k=1}^{M} \varsigma^{m k}\left(R^{k}, S^{m}\right) R^{k}\right],
$$

where $X^{m}$ is defined in equation 2.4 and $S^{m}$ is the sensory input for the m-th modality. This is the canonic equation that we are looking for. Here $\theta_{m}=\sum_{l} Q^{m}(l) \geq 0$, $\gamma^{m}=\tilde{\gamma}^{m} \cdot \theta_{m}$ and $\varsigma^{m k}=\tilde{\varsigma}^{m k} \sum_{l} Q^{m} Q^{k} \geq 0$. In the case when it is necessary to describe the interaction between $K^{m}$ different mental processes, for example, attention, perception and emotion that are represented by their own set of modes (c.f. 2.1):

$$
X^{m}(q, t)=\sum_{i=1}^{K^{m}} x_{i}^{m} \phi_{i}^{m}\left(q^{m}\right),
$$

where $\phi_{i}^{m}$ is the projection function of the $i$-th mode of the $m$-th modality. We have to use the generalization of the canonic model in the form:

$$
\begin{gathered}
\tau_{i}^{m} \frac{d x_{i}^{m}}{d t}=x_{i}^{m} \cdot\left[\sigma_{i}^{m}\left(R^{m}, S^{m}, C^{m}\right)-\sum_{j=1}^{K^{m}} \rho_{i j}^{m} x_{j}^{m}-\sum_{k=1}^{M} \sum_{j=1}^{K^{m}} \xi_{i j}^{m k} x_{j}^{k}\right] . \\
\tau_{i}^{m}=\sum_{q^{m}} \phi_{i}^{m}\left(q^{m}\right) .
\end{gathered}
$$

where $\rho_{i j}^{m}$ is the matrix of inhibitory connections between the modes of the same modality and $\xi_{i j}^{m k}$ is the connectivity matrix between the modes of different modalities. Here $\sigma_{i}^{m} \sim \tau_{i}^{m}, \rho_{i j}^{m} \sim \sum_{q^{m}} \Phi_{i}^{m}\left(q^{m}\right) \Phi_{j}^{m}\left(q^{m}\right), \sigma, \rho \geq 0$.

The hierarchical canonic model is based on a neuronal activity rate description. It can 
be generalized in the case of spiking neuronal ensembles (see (Nowotny and Rabinovich, 2007)). Both the rate- and the spiking- canonic models have in the corresponding phase space a new dynamical image - a stable heteroclinic channel (SHC) that implements robust sequential dynamics, which can describe a wide variety of cognitive activity. The necessary condition of its existence is the presence of nonsymmetry reciprocal inhibitory connections between neuronal groups that form the specific cognitive modes.

\subsection{Metastable states. Stable heteroclinic channels.}

Now we come back to the role of metastable states in cognitive dynamics. Why metastable states are so important? As imaging experiments shown (see, for example, (Sadaghiani and Kleinschmidt, 2013)), brain activity displays non-stationary dynamics as it reveals highly structured patterns across several spatial scales -from fine-grained functional constructions in sensory cortices to global brain networks. Such patterns can be interpreted as metastable states. Nature uses them in order to make transient cognitive processes structurally stable - robust against noise. One can explain the mechanism of such stability as we describe below.

A stable heteroclinic channel (SHC) is the only mathematical image in the phase space of rate or spiking canonic models that modern nonlinear dynamics can suggest for the representation and analyses of robust transient processing in the brain (Rabinovich et al., 2012b). Topologically a SHC is a vicinity of a heteroclinic chain that in fact is a sequence of metastable states, e.g. a chain of saddles connected by unstable separatrices as presented in Figure 3.
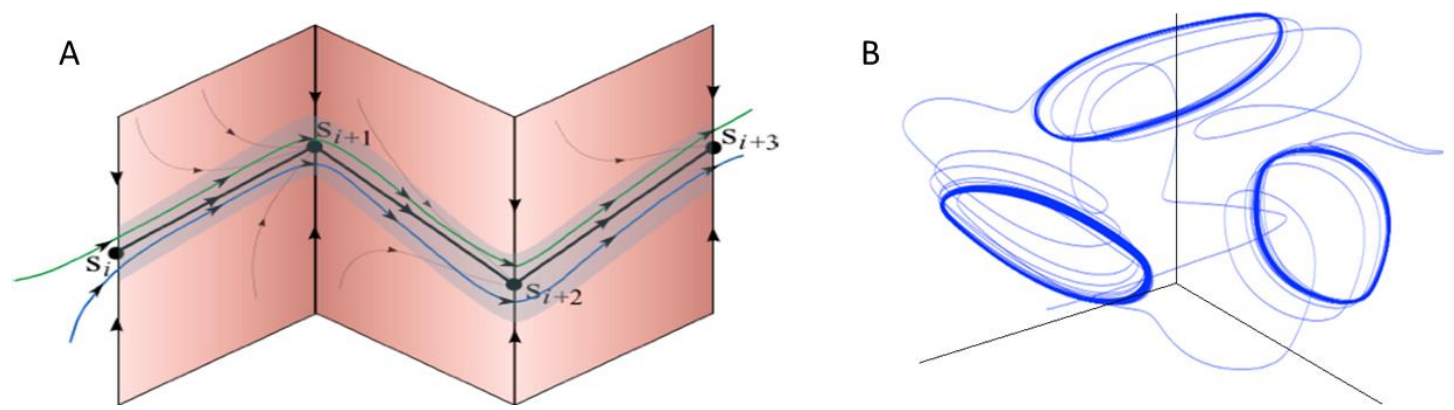

Figure 3 (Color online). Illustration of a heteroclinic channel in the phase space. A: sequence of metastable states representing the informational items, each metastable states is static - saddle fixed point. $S_{k}$ is the number of the item. B: sequence of dynamical metastable states - the mathematical image of these informational items in this case is a saddle limit cycle. Adapted from (Rabinovich et al., 2013).

Recent works have highlighted ways in which heteroclinic connections within attractors can be responsible for intermittent and switching behavior in complex nonlinear systems. Heteroclinic dynamics is an appropriate mathematical framework 
for transient processes that can be treated as an itinerary pass through metastable states (see (Huerta and Rabinovich, 2004; Rabinovich et al., 2008; Rabinovich et al., 2006b)).

\section{Stability and coordination dynamics of the sequences.}

\subsection{Finite information processing resources. Attention and working memory capacity - stability limit.}

The human brain cannot fully process the mountains of information that are received from external and internal worlds. Attention is the process that sequentially chooses the most important information for the performance of a specific cognitive goal. In fact, the limitation of such performance is a result of the interaction between capacitylimited content-specific information channels (short term -working-memory WM) and capacity-limited attentional processes (Marois and Ivanoff, 2005).

Our cognitive processing is not only limited by the rate at which information can be attended or consolidated into short term memory, it is also limited by the number of informational items that can be stored in this short time memory. Although the capacity of such memory is usually estimated to be about $5 \pm 2$ items (Baddeley, 1992), it is set not only by the number of objects or chunks but also by their complexity (Alvarez and Cavanagh, 2004). We define the capacity of sequential WM as the number of items that can be recalled correctly after a WM task. WM capacity varies amongst different individuals, depending also on age and health conditions (Edin et al., 2009; Oberauer and Kliegl, 2006; Rouder et al., 2008; Swanson, 1999).

Information processing related to WM activity can be represented in an abstract space (the phase space of the network that implements the working memory) as a continuous flow of a "liquid phase": the incoming information encoded in a spatiotemporal manner excites a specific network with a functional reverberate loop that sustains the corresponding information item for a finite time (e.g. based on a transient attractor like a SHC). The item networks are interconnected with each other in an inhibitory fashion and build a macroscopic network that keeps the whole sequence of items. The cooperative dynamics of this macro-network can be also based on the WLC principle and guarantee that the sequential items are recalled in the right order (Rabinovich \& Varona, 2011). This perspective has also been discussed in another model based on an echo state network (Pascanu and Jaeger, 2011).

Bick and Rabinovich showed that sequential WM capacity in the context of the discussed approach is limited by conditions of the retrieval process stability based on a model like (2.5) (Bick and Rabinovich, 2009). The stability conditions demand a 
sufficient level of inhibition in a WM random network and this level increases exponentially with the WM capacity. If we assume that the ratio between the level of mutual inhibition between the items and the self-inhibition $\rho_{i j} / \rho_{i i}$ is in the order of 20 (large enough from the neurobiological point of view), the number of successfully recalled informational items, according to the exponential law, is close to seven (see Figure 4). Recently, a fMRI study has provided experimental evidence for the dependence of the capacity limitation for visual WM on the level of inhibition (Edin et al., 2009). The stability condition of transient brain dynamics is a powerful tool for addressing and predicting several aspects of limitations of cognitive information processing.

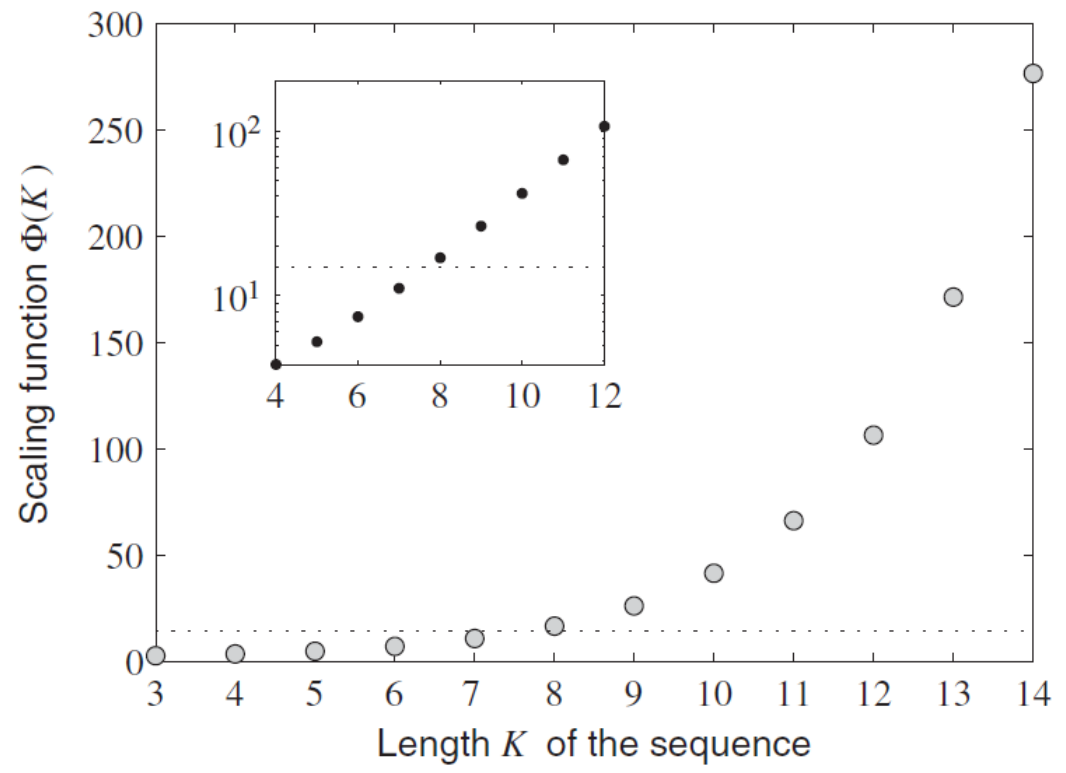

Figure 4. Dependence of sequential memory capacity on the normalized level of network inhibition $\Phi(K)=\rho_{i j} / \rho_{i i}$ (adapted from (Bick and Rabinovich, 2009)).

WM capacity depends on aspects related both to the environment and to the individual. In the discussed model (2.3)-(2.6), this is reflected in the model's architecture through subject and environment-dependent parameters, i.e., connection strengths that represent lateral inhibition.

3.2. Intrinsic attentional dynamics - sequence generation and the perception of the arrow of time.

As we already said, attention is not a static entity but a process. Such process can be based on a finite set of metastable states and, from a general point of view, has to demonstrate some kind of recurrence. Intrinsic attention dynamics is not necessary purely periodic and, as a working memory, is associated with several bands in the 
human brain rhythm spectra (Hsieh et al., 2011). Several experiments have emphasized that the mind segments dynamic experience into a discrete event representation (Zacks and Tversky, 2001). In particular, such discretization is typical for working memory and perception (Liverence and Scholl, 2012; Schwartze et al., 2011; Swallow et al., 2009). The segmentation of dynamic scenes is clearly related to attention (Buschman and Miller, 2010; De Freitas et al., 2012).

Remembering that the attentional network consists of several parts - components inhibitory interconnected, it is reasonable to hypothesize that attention rhythmicity has a winnerless competition dynamical origin. Using simple WLC networks, authors have demonstrated both rhythmic sequential switching of events and chaotic dynamics that nevertheless keep a temporal order along the recurrent process whose events demonstrate irregular time intervals (see (Varona et al., 2002; Venaille et al., 2005)). It is important that these sequentially changing discrete events can be differently labeled. In particular, different frequency of oscillations can be observed inside of different events (metastable states). Such attentional "clock" can possibly be used for the representation of the arrow of time, and the recognition and recall of the temporal structure of perceptual events (about the hierarchy of temporal perception see (Hasson et al., 2008; Kiebel et al., 2008)).

\subsection{Brain-to-brain temporal coordination.}

During social interaction partners have to coordinate their attention. Interbrain synchronization has been investigated during interpersonally coordinated actions (Hari and Salmelin, 1997; Konvalinka and Roepstorff, 2012; Müller et al., 2013; Sänger et al., 2013, 2011), particularly in the context of tasks such as imitation (Dumas et al., 2011, 2010), card playing (De Vico Fallani et al., 2010) and playing instruments in a music ensemble (Lindenberger et al., 2009; Müller et al., 2013; Sänger et al., 2013, 2012). Such synchronization can be related to the interacting representations of the individual's own actions and his/her partner's actions. These representations are presumably formed to achieve temporally aligned activity modulation (Lindenberger et al., 2009; Sänger et al., 2011). A particularly clear example is represented by ensemble musicians, who presumably achieve and maintain synchrony by emulating each other's concurrent and predicting each others' future actions (Rasch, 1979; Keller et al., 2007). Such kind of coordination can happen under correlated mutual stimuli actions. For example, when two violinists play together they coordinate the rhythm and melody by mutual auditory-sensory signals. Based on our model of intrinsic attentional dynamics (see (2.3)), we analyzed this coordination process. We keep in mind that to describe the dynamics of each player we need to use different networks that represent separately rhythm and melody (Lappe et al., 2013).

We simulated the interaction between two 6-element networks described by (2.5) 
with bidirectional excitatory connections for 3 elements which we will call $B, C$ and $\mathrm{E}$ and its corresponding elements in the second network. The parameters for these networks are described by [P1-P4] in the appendix 7.2.

The excitatory connection between networks was determined by the value of the parameter $\xi$ (see Figure 5). We have observed a very strong coordination of two brain attentional networks with moderate values for the excitatory connections close to $\xi=0.5$, see Figure 6.

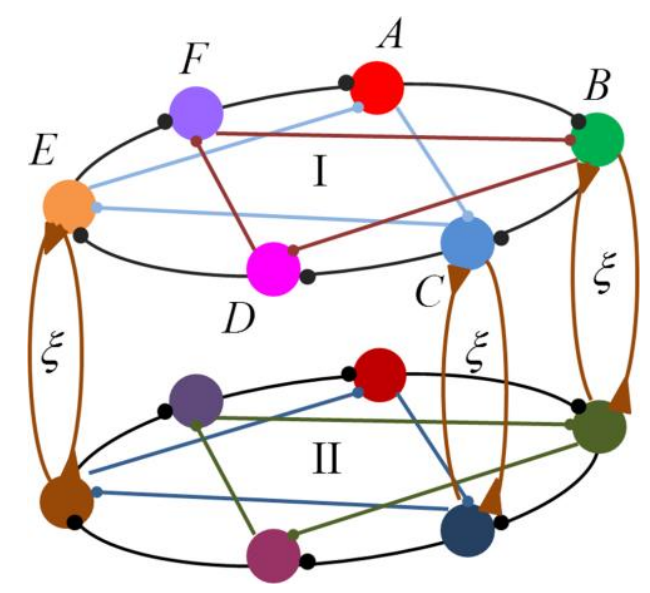

Figure 5 (Color online). 6-element attentional networks, I and II, interacting by means of an excitatory connection $\xi$ between the corresponding elements B, C and E. 


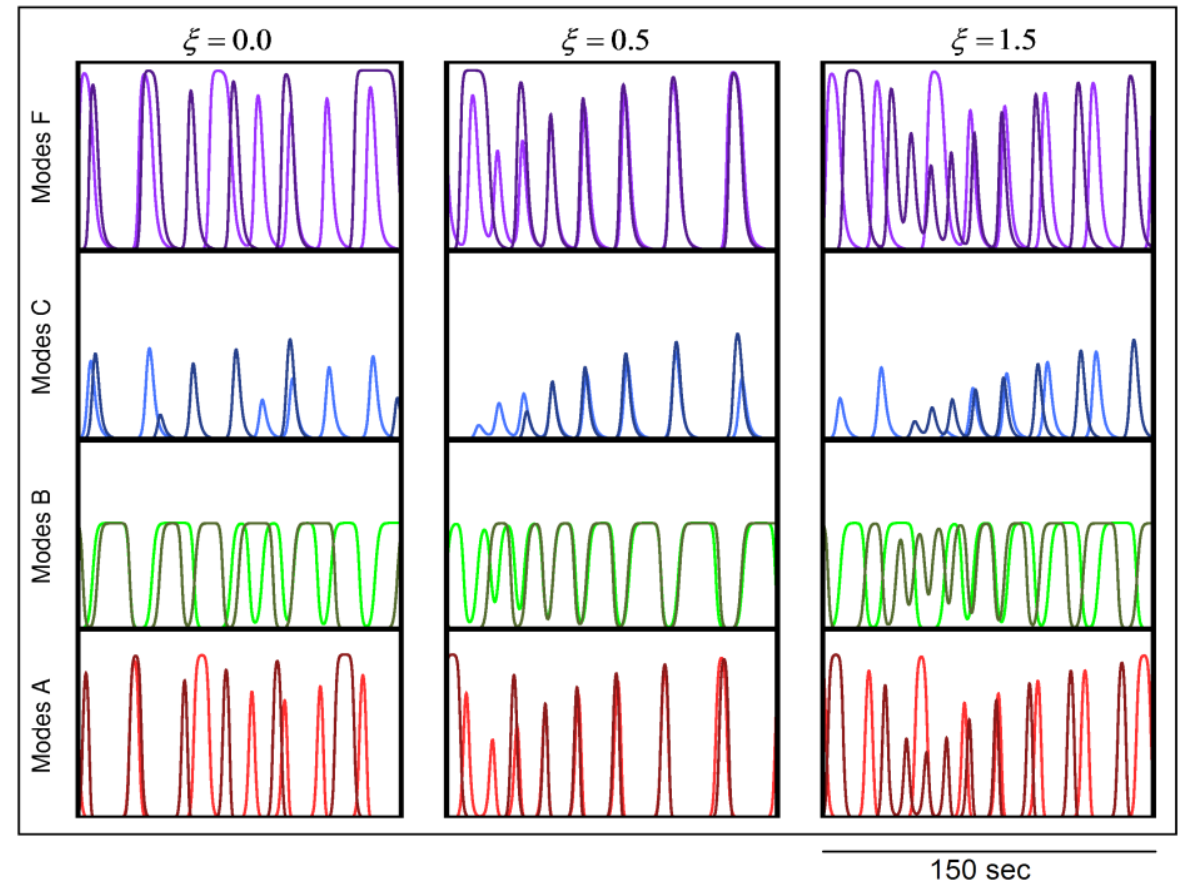

Figure 6 (Color online). Effect of the variation of the strength of the excitatory connection $\xi$ among the corresponding elements B, C and E in two attentional networks I and II by portraying the 4 most active elements in each case for $\xi=0.0$, $\xi=0.5$ and $\xi=1.5$ during the same period of evolution (from $t=100 \mathrm{~s}$ to $t=250 \mathrm{~s}$ ). Network I activity is represented by light colors and Network II activity is represented by dark colors.

We interpret these results in the context of the dynamical coordination of brain-tobrain coupling in terms of the attentional processes focused on a joint cognitive or behavioral task. Clearly, a too strong interaction between two brains can destroy their individuality and in fact create a new complex system. On the other hand, the described model represents sequential activations which occur in a history-dependent manner. Thus, in principle it could be used for the interpretation and prediction of interpersonal action coordination.

\section{Dynamics of attention sharing, switching and decision making}

\subsection{Low-dimensional mind dynamics}

We analyze here the dynamics of attentional inhibitory networks together with sequential multimodal mental activities in the framework of a global mode interaction approach (Rabinovich et al., 2008; Rabinovich and Varona, 2011; Rabinovich et al., 2008; Rabinovich et al., 2012a). The results presented below, invariant to different temporal scales, are based on the discussed principles in attentional dynamics, mainly 
the principle of limited capacity of information processing. To investigate the dynamical mechanisms behind the cooperative activities of different modalities under attentional control, our general model of focused attention is implemented based on both bottomup and top-down information flows (see Figure 7 and also (Lee, 2008)). In this figure, modes $\mathrm{R}^{\mathrm{i}}$ represent metastable states that change sequentially in time and the term modality $\left(\mathrm{X}^{\mathrm{i}}\right)$ refers to the different cognitive, emotional or sensory processes.

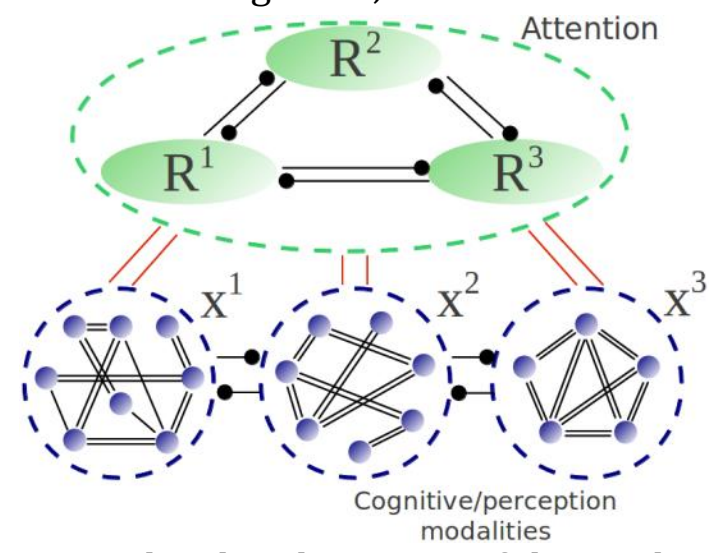

Figure 7 (Color online). Hierarchical architecture of the mode interaction in the case of three modality processing $\left(\mathrm{X}^{1}, \mathrm{X}^{2}, \mathrm{X}^{3}\right)$ whose interaction is described by systems (2.42.5). The upper level of the hierarchy represent the attention network. Attentional modes $\mathrm{R}^{1}, \mathrm{R}^{2}, \mathrm{R}^{3}$ correspond to different modalities (cf. equation 2.3). Black circles mean inhibitory connections.

In (Rabinovich et al., 2013) we have used the canonic excitatory-inhibitory model (2.3), (2.5) of an attentional network (in the case of nonsymmetric inhibitory connections) that satisfies the information processing stability principle. In the general case for $m>2$, after a short transient period, all system's activity takes place in the vicinity of the unstable separatrices of the metastable states. We will show this for the case $m=3$ in this section (a general enough case for a realistic number of modalities that dynamically share attentional resources).

The analysis of model (2.3) shows that key transformations of attentional dynamics can occur in a restricted area of the phase space. This is illustrated in Figure 8 for the case of three interacting modalities. As time increases all trajectories are attracted by a quasi-two-dimensional volume. This volume is the vicinity of a 2-D surface that is named simplex (for a rigorous definition see (Zeeman and Zeeman, 2003)). On the other hand, the simplex itself has a finite size limited by boundary separatrices (see Figure 9). Thus, all robust attention activity is described by the trajectories, which after a short transient are located in a low dimensional finite volume. In the informational phase space, this finite volume represents the limitation of information processing resources. 


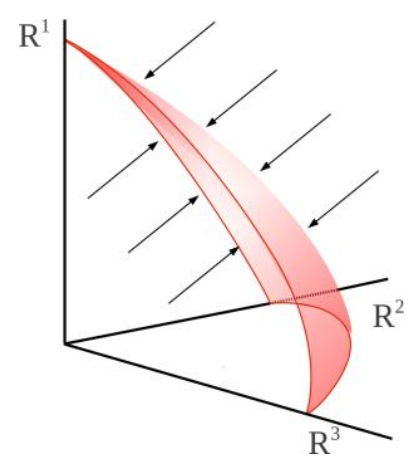

Figure 8 (Color online). Representation of a simplex in a 3-D competitive system (2.3). Adapted from (Rabinovich et al., 2013).

Attention control strategies are formed during development and learning stages. Figure 9 shows different cross modality dynamics that can be studied in the context of the discussed model. The selection of the strategy depends both on the cognitive task and the environmental conditions. This is formally expressed in the model (2.3) by the value of the excitation level $\sigma_{i}^{m}$ of specific attentional modes and the competition between them $\varsigma^{m k}$. In particular, in the case of strongly competitive and equivalently important tasks, these values are $\varsigma^{m k} \sim \varsigma^{k m}$ and $\varsigma^{m k} / \varsigma^{m m}>1$. Depending on the initial conditions, attentional resources can be focused just on one of the modalities. This type of dynamics is named multistability (see Figure 9A). When different modalities are not equally balanced in the competition (e.g. non-reciprocal competition) another kind of multistability arises. In this case, the dynamics of $R^{1}, R^{2}, R^{3}$ can become much richer. In particular, the stable fixed point (focus in 9B) turns into an unstable point and, as a result of a Hopf bifurcation, a stable limit cycle appears (9C). Another possible situation consists in the coexistence of a stable fixed point and a stable limit cycle (see 9D). In this case, a short external stimulus can change the attentional control strategy: instead of a static distribution of attentional resources (stable fixed point in 9D), the system performs a rhythmic modulation of the cognitive activities (stable limit cycle in 9D), see also Figure 10. 

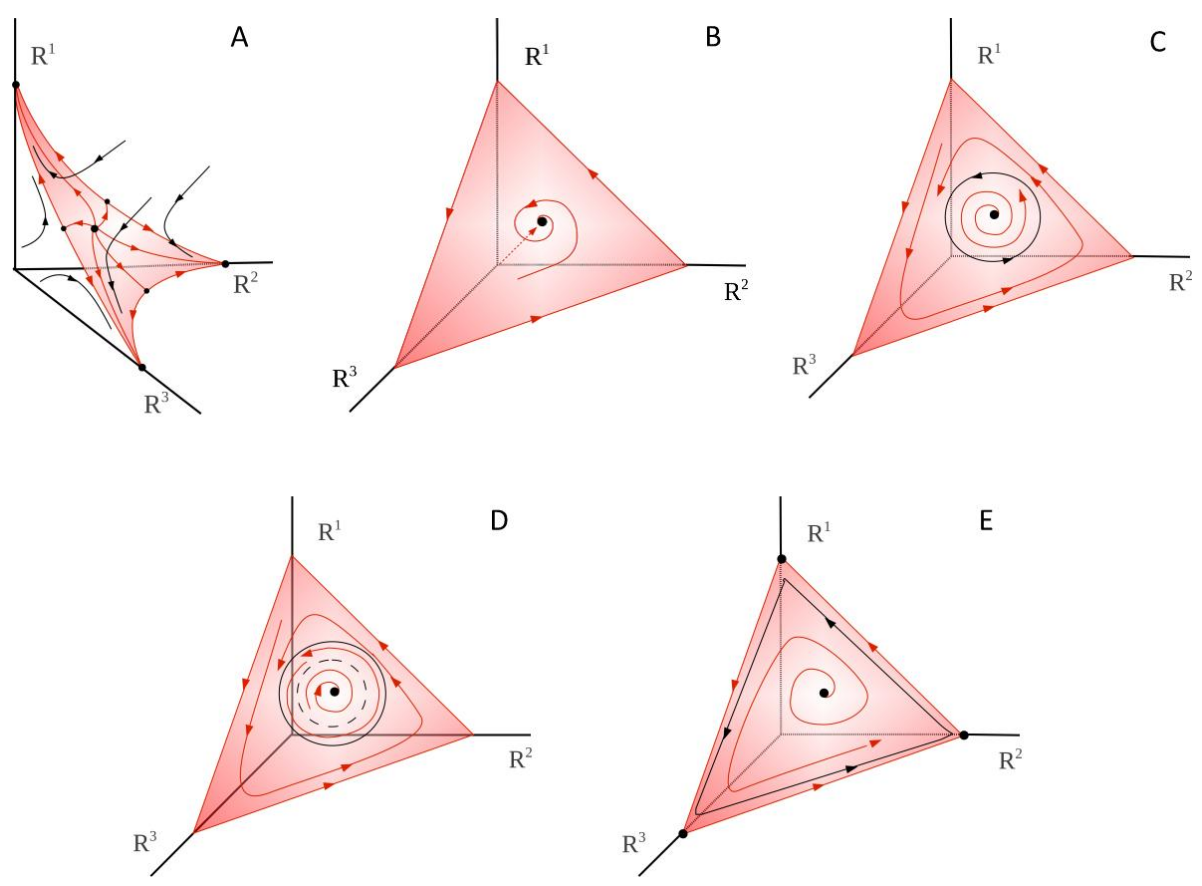

Figure 9 (Color online). Crossmodality attentional dynamics in model (2.3), $M=3$. Panel A corresponds to the case in which complete attention is focused on one of the tasks multistability on the simplex - three stable fixed points whose basins of attraction are bounded by separatrices of the saddle fixed points. Panel B -multitasking- corresponds to the coexistence of cognitive tasks that just need partial attention each - the image is a stable fixed point that is a global attractor on the simplex (c.f. Figure 8). Panel C shows that a stable limit cycle emerges on the simplex, this is the mathematical image of periodic changes of attention levels. Panel D represents bistability - the coexistence of two attractors, i.e. stable fixed points and stable limit cycle. Finally, panel E shows a stable limit cycle in the vicinity of a closed heteroclinic contour which represents the sequential switching of attention among three different modalities. For the mathematical details of these bifurcations see (Zeeman, 1993). It is interesting that, under rhythmically changing stimuli, the attention switching between different modalities can become irregular, see Figure 10, which can be related to irregular perceptual alternations (Dieter and Tadin, 2011). Adapted from (Rabinovich et al., 2013). 


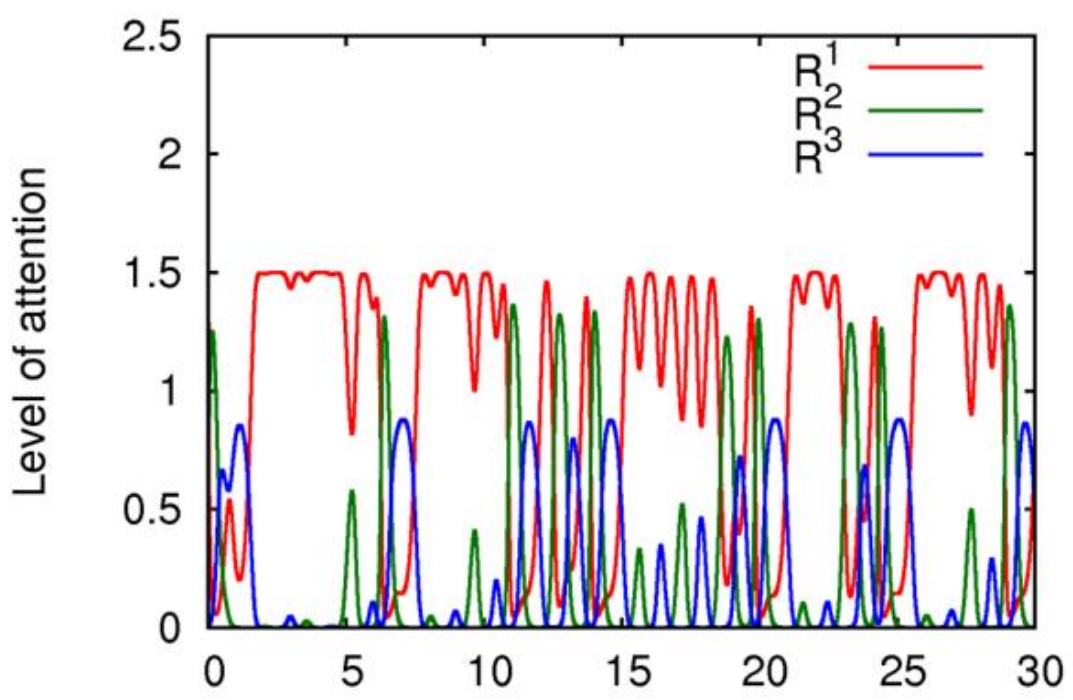

Figure 10 (Color online). Irregular attention switching under the action of a periodic stimulus on the first modality. The parameters $\varsigma^{m k}$ used in this simulation are indicated in [P5], see appendix 7.2. Adapted from (Rabinovich et al., 2013).

\section{2. $\quad$ Attention sharing and perception binding}

Multi-electrode recordings from different areas of sensory systems in several animal models have shown that sensory information on the first level of processing is encoded as spatio-temporal patterns by networks that implement a winnerless competition (WLC) interaction of different dynamical modes which leads to their sequential switching in time (see (Jones et al., 2007; Mazor and Laurent, 2005; M. Rabinovich et al., 2008). In the phase space, such sequential dynamics can be represented by a stable heteroclinic channel (see Figure 3).

In many cases, to make the representation of environment faster, more robust and reliable, the brain may use the binding principle - sharing the attention between different sensory modalities in order to integrate different sensory information on the lowest level of hierarchy (Anderson et al., 2010; Singer, 2013). Motivated by the multisensory attention dynamics observed in neural systems, authors in (Rabinovich et al, 2010) built a model that describes the heteroclinic integration of channels representing different modalities (binding dynamics). This model is valid in the assumption that the different attentional modalities do not change in the binding process (stable fixed point in the simplex, see Figure 9b). The model is described by equation (2.5) with $M>1$. 


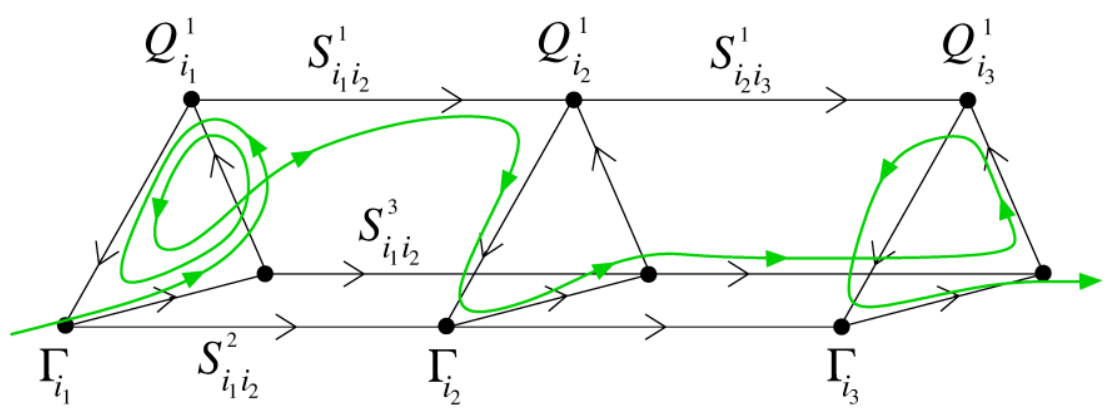

Figure 11 (Color online). Illustration of a multimodality attentional channel and a trajectory corresponding to the binding activity, $\boldsymbol{Q}$ and $\Gamma$ represent the metastable states and $S$ are the corresponding separatrices (adapted from (Rabinovich et al., 2010)).

The theoretical and computational analyses regarding heteroclinic binding in (Rabinovich et al, 2010) can be summarized in two main points: (i) for a wide range of control parameters, i.e., the levels of excitation and inhibition, in the phase space of the model, there exists an object named a multimodality heteroclinic channel, and the trajectories in the vicinity/inside of this dynamical object represent an integrated (binded) information flow of different modalities (see Figure 11); (ii) the time series and spectrum of these multimodality-trajectories demonstrate relevant features mutual modulation and regularization of the different modalities and, correspondingly, the appearance of new components in the power spectrum. It is important to note that the proposed dynamical mechanism for binding accounts for different levels of temporal hierarchy, ranging from milliseconds to minutes. The information about the recognizable image coding by such modes could be transferred to the cognitive and behavioral modes for the next processing. This phenomenon is in principle suitable to be analyzed in fMRI data, see (Raizada and Kriegeskorte, 2010).

\subsection{Information flows in the phase space.}

To quantitatively characterize the effectiveness of the heteroclinic attentional binding, let us introduce a function called Information Flow Capacity $\left(C_{I F}\right)$ as

$$
C_{I F}(L)=\sum_{l}^{L}\left(\Delta C_{I F}(l)\right)
$$

where

$$
\Delta C_{I F}(l)=J_{l} \sum_{j=1}^{J_{l}} \frac{\lambda_{j}^{l}}{\left|\lambda_{J_{l}+1}^{l}\right|}
$$


Here $l$ is the number of metastable states (saddles) along a channel at time $t_{L}$, when the system has reached the saddle $L, \lambda_{j} l$ are the Lyapunov exponents of saddle $l$, and the integer $J$ is defined by the conditions (see also appendix 7.1):

$$
\sum_{j=1}^{J} \lambda_{j}>0, \sum_{j=1}^{J+1} \lambda_{j}<0
$$

If the unstable separatrices of all saddles along the heteroclinic channel are onedimensional, we have

$$
C_{I F}(L)=\sum_{l}^{L}\left(1+\frac{1}{v_{l}}\right)
$$

since all $J_{l}=1$, and the ratio of positive Lyapunov exponents to the negative ones is just equal to the saddle value $v_{l}$ in degree -1 . We have to remind here that for the channel stability the product of all saddle values has to be larger than one, see (Rabinovich et al., 2012; Rabinovich et al., 2010a).

$C_{I F}$ is a useful meaure for a quantitative description on the example of heteroclinc binding problem. As can be observed in Figure 11, each saddle along a "binding heteroclinic network" has two unstable separatrices. That means all $J_{l}=2$. Thus, the estimation of $C_{I F}$ informs us that the flow capacity for a binding channel is at least two times larger than the $C_{I F}$ of three independent heteroclinic channels. In this way, the information flow capacity can serve to characterize the complexity level of the trajectories within an attentional channel network.

\subsection{Decision making strategy.}

There are two different processes that researchers name 'decision making': (i) a fast switching of the cognitive state in the mind phase space, and (ii) the controlling of cognitive parameters, in particular functional connections in attentional networks, in order to trigger the attentional strategy from one type to another. The first group of decision making processes is illustrated by Figure 12A - a short sensory or emotional impulse enables to move the system from the basin of attraction of one limit state to the basin of attraction of another. Noise, of course, can make such process unrealistic because it may destroy the boundaries between different attractor basins in the multistability case. In contrast, strategic decision-making can often be viewed as decision-making under uncertainty. Such decision making involves the attention of goal formulation, problem identification, and alternative generation, evaluation and selection (Schwenk, 1984).

Let us consider a man driving a car, half-listening to the local news on the radio and simultaneously receiving a call on his cell phone. Suddenly, he hears a message on the radio of a car accident just a mile ahead. A newscaster is describing the situation to the 
drivers in the vicinity of the accident to help them figure out a way to avoid the accident-affected area. The driver has to react accordingly: first to turn off his cell phone, and then stop the car before planning a new route by concentrating on the newscaster advice. This sequence may be repeated from time to time. We can describe such behavior modification in the framework of the hierarchical dynamical model (2.3), (2.5). The multitasking behavior before the driver gets the message on the radio is the regime of the divided attention. Such regime is represented in the phase space of (2.3) by the stable fixed point on the simplex (see Figure 12A). When the newscaster announcement starts, the parameter $C^{m}$ (for simplicity suppose $m=1$ ) makes the inhibition of modes $R^{2}$ and $R^{3}$ by $R^{l}$ stronger $\left(\varsigma^{21}, \varsigma^{31}\right.$ become larger). One can see the corresponding global bifurcation in Figure 12B-instead of a multimodal fixed point, the system is represented by just one global attractor: a stable node on the axis $R^{l}$. Such kind of dynamics is usually labeled as 'winner-take all' (WTA). It corresponds to the focusing of full attention on the performance of just one task - deciding a new driving route based on the newscaster advice and the driver's previous knowledge and experience. The two other modalities $X^{2}$ and $X^{3}$ are just suppressed because they have no excitation: $\sigma_{i}^{m}=\sigma_{i_{0}}^{m} R^{m}-q_{i}<0,(m=2,3)$.

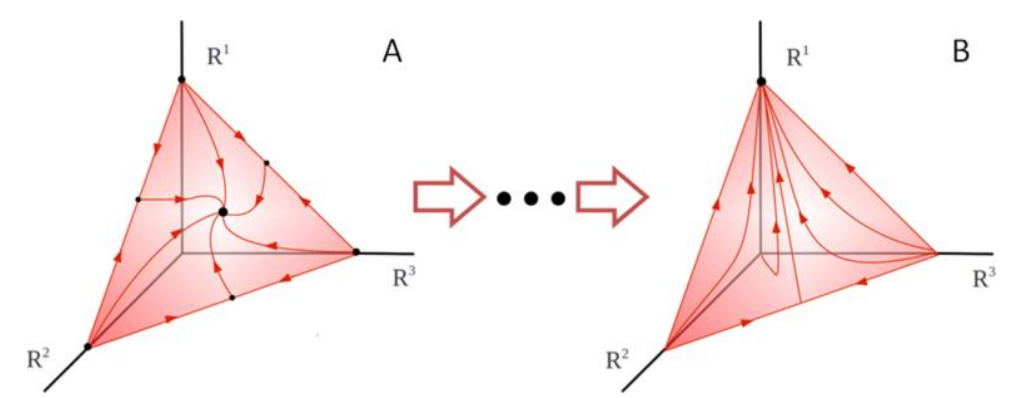

Figure 12 (Color online). The transition from the case of shared attention (A) to a winner-take-all attention regime (B), as observed in the dynamical system (2.3), $M=3$.

A strategic decision making means choosing a functional structure of the interactive modes by changing the set of exciting neuronal clusters and their inhibitory connections (the architecture of the functional networks that depends on the stimulus). The overlapping of the attention and decision making networks is well established. For example, parietal neurons encoding saccade motor decisions also carry signals of attention (perceptual selection) that are independent of the metrics, modality and reward of an action. Gottlieb and Balan have proposed that attention implements a specialized form of decision based on the utility of information (Gottlieb and Balan, 2010). Oculomotor control depends on two interacting but distinct processes: attentional decisions that assign value to sources of information and motor decisions that flexibly link the selected information with actions (Gottlieb and Balan, 2010). 
In the discussed model, attention can be mediated by changing the dynamics of competing multi-modal sensory representations. This can be regarded as changing the attractor manifold in a state dependent fashion - in a way that depends upon the states of the different attentional parts of the hierarchical network. This necessarily induces a separation of temporal scales, in the sense that the fast dynamics at lower levels are governed by slower changes in attentional dynamics - which themselves respond to switches in cognitive sets. The theme of state dependent changes in control parameters fits well with recent proposals of how attention is mediated during hierarchical Bayesian inference or predictive coding. In these models, the attentional modes change interactions among neuronal states in low levels through modulating postsynaptic gain, which encodes the precision of sensory information (see also (Feldman and Friston, 2010)).

\subsection{Attention - pain interaction.}

It is well known that pain influences attention and cognition (Eccleston and Crombez, 1999; Van Damme et al., 2010). We all experience inability to concentrate when suffering pain. Even weak pain can crucially change perception or cognition. Let us analyze the effects of moderate pain on the simultaneous perception of three different sensory modalities, for example, visual, auditory and olfactory. As we discussed above, the brain shares finite attentional resources to process these informational signals resulting in resources proportionally allocated to these modalities. Based on the dynamical model (2.3), (2.5), we can interpret the effects of pulsating pain. The pulsating (throbbing) pain is typical, for example, for patients with chronic migraine (Ahn, 2012; Goadsby, 2013). In general, recent experiments indicate that the throbbing quality is not a primary sensation but rather an emergent property of neural networks within the central nervous system (Mirza et al., 2012). In fact, the dynamics of such networks reminds the dynamics of central pattern generators (see (Yuste et al., 2005)).

Throbbing pain does not necessarily interrupt the cognitive processing but qualitatively changes its dynamics. In particular, our modeling shows that the dynamics of attention distribution among the participants of the information processing can become chaotic under the action of such pain. Of course, the pain-attention dynamical interaction is reciprocal. In particular, focused attention and distraction enable the modulation of the perception of pain intensity (Rabinovich et al., 2012). In (Chan et al., 2012) authors have shown that the brain enables to shift attention to a self-generated sub-nociceptive image and to rehearse it. Event-related potentials were used to study the possible processes associated with a focus-then-orient attention strategy. Focusthen-orient attention across nociceptive and sub-nociceptive images appears to be related to response inhibition. Such modulation seems to be mediated by generating 
and maintaining sub-nociceptive images in working memory. The sequential focusthen-orient processing of somatosensory images incorporates: (i) the focus-attention strategy, which places attention on the nociceptive stimulus felt; and (ii) the distraction strategy, which switches attention to self-generated sub-nociceptive images and rehearses them (see also (Woolf, 2010)).

The neurophysiological mechanisms behind the interaction of attention and working memory with pain are currently analyzed using functional magnetic resonance imaging. In particular, in (Bingel et al., 2007) Ulrike Bingel and coauthors have shown that the source of modulation for working memory could be attributed to the parietal cortex, the modulatory effect of pain was observed in the rostral anterior cingulate cortex, an area suited to link pain perception and attentional control.

\section{Attentional hierarchy: Creativity and disorders}

\subsection{Attention - emotion hierarchical dynamics. Mental disorders.}

To describe the interaction between attention and emotion, Rabinovich and coauthors (Rabinovich et al., 2010b) introduced a hierarchical dynamical model that consists of just two competitive attentional modalities - cognitive and emotional. However, each of them is characterized by several intrinsic modes and thus it demonstrates complex dynamics. Mathematically, the structure of this model is similar to equations (2.3), (2.5).

Obsessive-Compulsive Disorder (OCD) is a very common anxiety disorder. Obsessions are defined as persistent thoughts, impulses or ideas that are experienced as inappropriate and that generate anxiety or distress. On the other hand, compulsions are defined as repetitive actions that are often done against the will of the subject - rituals (e.g., compulsive hand washing) or mental acts (e.g., silently repeating numbers) that are typically performed in an attempt to relieve the distress brought on by the obsessions. The fast attention switching from the performance of a cognitive task to the ritual is a dynamical phenomenon that characterizes individuals with OCD (see (Muller and Roberts, 2005)).

To address the hierarchical mental instability that is the main feature of an OCD subject, authors in (Bystritsky et al., 2012; Rabinovich et al., 2010b) have modeled the dynamics of an attention network (2.3), (2.5) in the region of control parameters where a sequence of metastable states - sequence of saddles - is characterized by instabilities with two different time scales. In particular, they investigated the case when one of these unstable separatrices leads to the next cognitive metastable state along a cognitive sequence, whereas the second unstable separatrix targets the emotional saddle that represents the entry to the ritual, which is modeled as a different stable chain of the "emotional" metastable states. The ritual terminates at a saddle that has 
many unstable separatrices, each yielding to a cognitive mode (see Figure 13). As a result, the OCD dynamics is represented by a transient in a $(N+M)$-dimensional space. Such transient qualitatively distinguishes itself from the normal behavior and from other disorders characterized by a specific instability that leads to uncertainty. The analysis of such modeling predicts that in OCD the interaction of the sequential cognitive activity (e.g., sequential decision making) with emotion is characterized by an intermittent dynamical instability. The corresponding dynamical attractor is represented in Figure 15 (Rabinovich et al., 2010b).

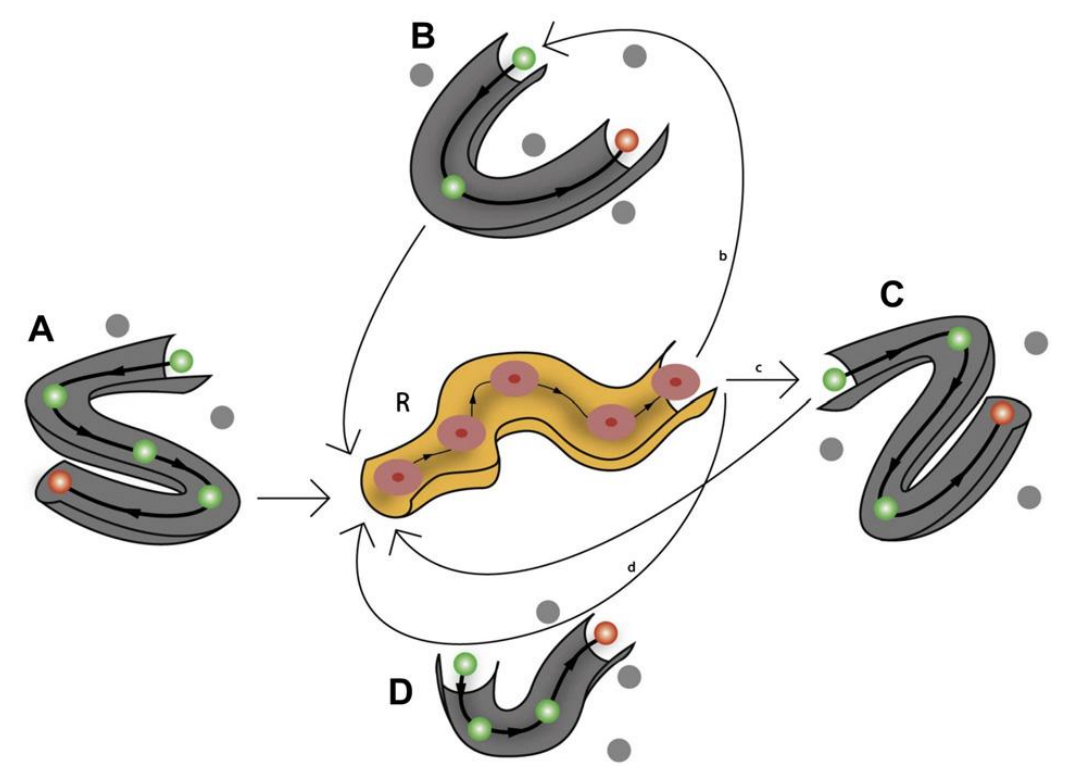

Figure 13 (Color online). Dynamical image of obsessive compulsive behavior. Gray balls represent metastable states in mental space which did not become active, green balls represent the SHC metastables states, and red balls represent the end of the activity (adapted from (Bystritsky et al., 2012)). The yellow channel represents the ritual, while the gray channels represent the goal-focused cognitive activity (see details in the text).

Figure 13 illustrates an unusual temporal feature - an abrupt onset of pathological thinking leading to repetitive behavior. The SHC labeled as A describes four steps (metastable states, represented by green balls) of a normal behavior, e.g. of writing with a pencil (i.e. picking up the pencil, carrying it to the piece of paper, placing it on the paper, etc.) leading to the final completion of the activity denoted by the red ball. However, in the case of someone with OCD, at the fourth step the person may suddenly realize that the pen is contaminated and interrupt the behavior in order to wash his/her hands (yellow SHC "R" ritual consisting of five additional steps such as picking up the soap, turning on the water, etc.). In the case of OCD, at completion of $\mathrm{R}$ the behavior may not return to the original state $A$ and instead could exit into several other possible SHCs representing different degrees of impairment dictated by emotion- 
cognitive appraisal of the situation. In case "B" the person could pick up the pencil and at the second step realize that the pencil is still dirty and then go back to the ritual of washing (SHC R). In the example of SHC (C), the person may have touched the pen and immediately returns to ritual (R). SHC (D) represents a state in which the person with OCD, once his/her symptoms are triggered, stays in the ritual without going back to the contaminated pencil until the resources are exhausted or some other factors come into play. Based on the discussed general model of attention-emotion instabilities, Bystritsky et al. have suggested a new methodological paradigm for diagnosis of various mental illness (Bystritsky et al., 2012).

Bipolar disorder (BPD) is another example of mood instability. This pathology is characterized by interruptions of the normal mood and the cognitive performance by episodes of elevated mood (e.g. euphoria) interspersed with episodes of depression. A typical mood time series of BPD is represented in Figure 14. Usually the focus of attention in each of these episodes is different (see (García-Blanco et al., 2013)).

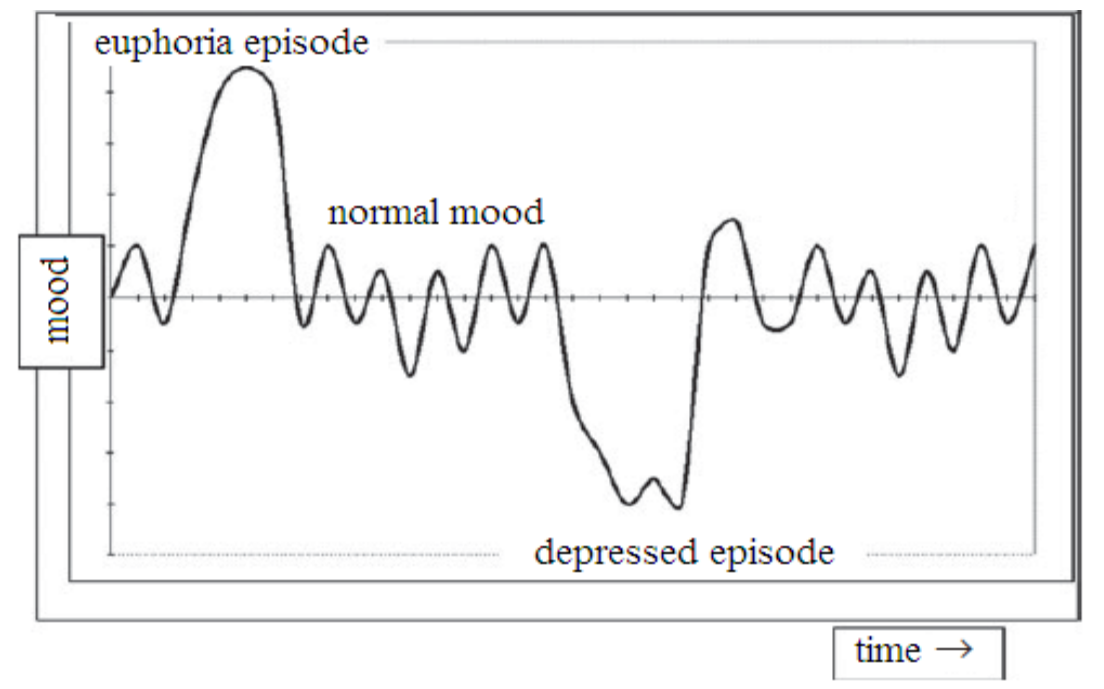

Figure 14. Typical temporal mood pattern in bipolar disorder. Adapted from (Bonsall et al., 2012).

In the framework of the model (2.3), (2.5) with 10 interactive modes -two attentional modalities (cognition and emotion), five cognitive modes, and three emotional modes (euphoria, normal and depression), Rabinovich and Muezzinoglu have proposed a dynamical image of bipolar disorder (Rabinovich and Muezzinoglu, 2010). This model can generate the sequential switching dynamics representing the mood alternation observed in the experiments (Bonsal et al., 2012), see Figure 15. 


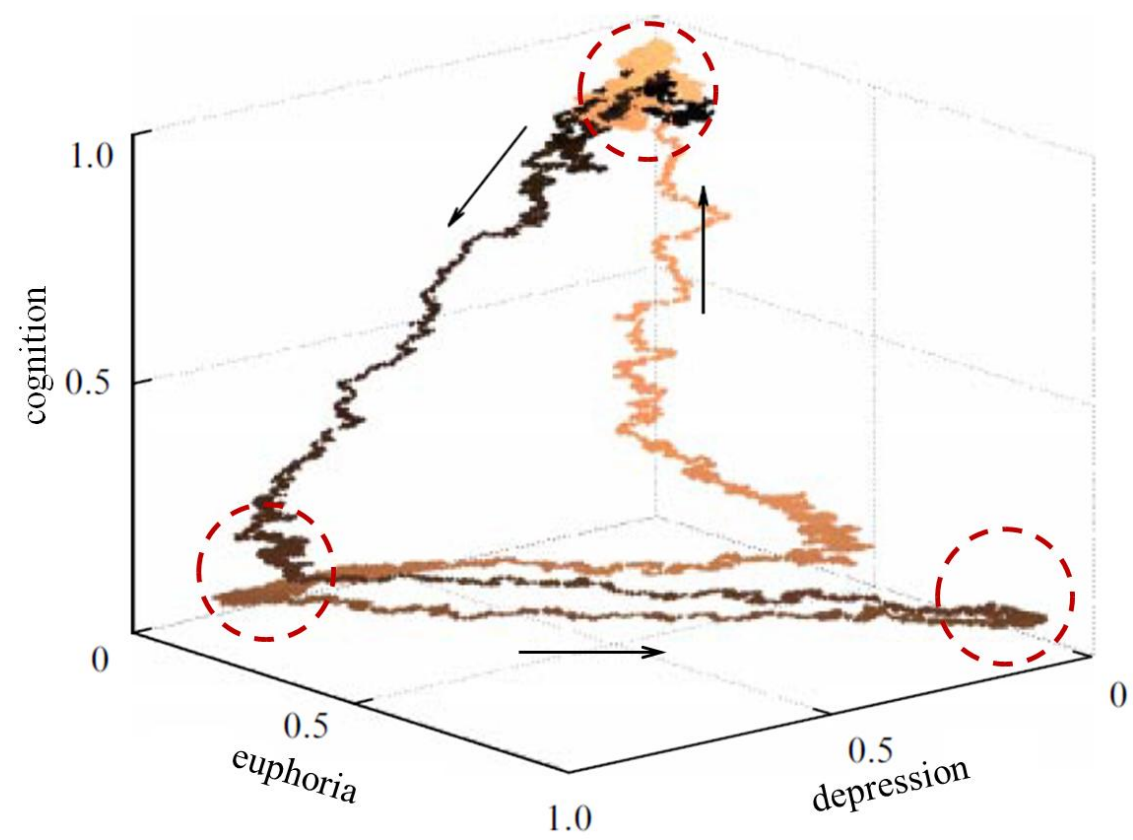

Figure 15 (Color online). Projection of the 10-mode sequential BPD model dynamics in a 3D phase space - vertical axis represents the joint activity of all five cognitive modes, and the two other axes represent two emotional modes -euphoria and depression, resulting in a sequential mood pattern. The dashed circles indicate the vicinity of the metastable states. Adapted from (Rabinovich and Muezzinoglu, 2010).

\subsection{Creativity: Attentional control turn off?}

As it can be deduced from the popular statement: "some animals may have larger brains than humans, but none has abilities of formal logic, self-consciousness, and creativity of man", to understand the dynamical origin of the creativity and the corresponding role of attentional control is a very challenging goal. In the last few years, several provocative experiments with imaging analyses have addressed the correlation of brain activity with creativity processes. We discuss here some of the results related to music and lyric improvisation. Based on experimental evidence one can say that attention influences creativity. In fact, creativity is the process of random playing with ideas or divergent thinking. This influence happens in two opposite ways: attention has to be distributed -non focused when new sequences of ideas are generated; at the same time attention needs focus when each idea is structured and performed. We will model here the dynamics of the core of creativity processes -the generation of new information that is related to a cognitive instability (Rabinovich et al., 2012a).

About a century ago Henri Poincare formulated the main principle of creativity: "The genesis of mathematical creation is a problem which should intensely interest the psychologist. It is the activity in which the human mind seems to take least from the outside world, in which it acts or seems to act only of itself and on itself, so that in 
studying the procedure of geometric thought we may hope to reach what is most essential in man's mind" (Poincaré, 2009). The generation of a new sequence of ideas is the result of the intrinsic activity of the mind, or in another words, the result of an instability developed and the generation of new sequences. Limb and Braun studies of jazz improvisation indicate that spontaneous improvisation, independent of the degree of complexity of the music, is characterized by deactivation of lateral regions of the prefrontal cortex together with focal activation of the medial prefrontal cortex (Limb and Braun, 2008). This unique pattern may offer insights into cognitive dissociations that might be intrinsic to the creative process: the innovative, internally motivated generation of novel material that can apparently occur outside of conscious awareness and attentional control. To build a dynamical model of these processes, it is important that "novel material" -the product of improvisation- is rule-based and structured. While the role of technique in freedom, expression, and improvisation is often disregarded, it is absolutely essential for the creativity process. For example, without the understanding of words, meaning and language structure, a poet cannot write a poem.

Experiments have shown that problem-solving, conscious self-monitoring and focused attention are suppressed in the main part of the improvisation processes (Limb and Braun, 2008). The opinion that spontaneous composition relies to some degree on intuition, the "ability to arrive at a solution without reasoning", supports the dynamical view on creativity as a process governed by an intrinsic cognitive instability that produces new information.

When we talk about a cognitive instability in general, it is important to remark here that creativity and mental disorders often go together. In particular, bipolar disorder, characterized by extreme mood swings of mania or euphoria to depression, has been associated with creative individuals, as mad virtuosi, geniuses or artists. Nevertheless, the relationship between bipolar disorder and creativity is not clear. Many psychiatrists agree that both bipolar disorder and creativity are related to the mental instability of the person and may have the same genetic origin (Murphy and Sahakian, 2001; Santosa et al., 2007; Strong et al., 2007).

Recently, the authors of the paper (De Manzano et al., 2010) have investigated the origin of the correlation between mental instability and creativity. They analyzed the role of dopaminergic transmitters (D2) that are associated with symptoms of schizophrenia and bipolar disorders. The experiments support the hypothesis that D2 plays an important role in human creative thought and behavior. The structural instability of cognitive dynamics as a precondition of creativity in the arts and science has been discussed by many authors, for a recent review see (Ansersson et al., 2013). We present here a model of the dynamics of the core of creativity process - the generation of new information that is directly related to an intrinsic cognitive instability in the context of the above discussed approach (Rabinovich et al., 2012a). 
Let us consider, as an example, information creation in the processes of jazz improvisation by a group of six jazzmen using drums, trumpet, saxophone, guitar, bass and piano. There are two different ways to create a new composition by six: the creativity can be a result of the cooperative instability in the "collective mind" of the team, or the new music can emerge when each of the partners is improvising with the same melody and harmony as a soloist - playing with different improvisation ideas. We will focus on the later. We name such action "jazz 6 sequential solo". Sequential switching between different soloists activity is guaranteed by the dynamics of attentional networks that consist of six modes connected in a WLC manner. For the sake of simplicity we suppose that the attentional networks dynamics of all partners are completely coordinated (synchronized) by mutual auditory excitations between all of them and thus it is possible to build a model as it is presented in Figure 16 (for details about the coordination and synchronization of WLC networks dynamics see (Rabinovich et al., 2006a; Venaille et al., 2005)). In reality, their attention does not follow an on-off dynamics - the "cost in time for the switching task" is finite and thus the activities of different jazzmen will overlap.

We also take into account that, when improvising ideas, working memory capacity is finite. Suppose all musicians use just six of such ideas - to scale sequential fragments, play off the melody ("ad-libbing"), change direction, skip notes or zig-zag, repetition, well-known solo within one's own solo (see (Reeves, 2006)). These ideas or informational items form creative mode networks in the mind of each player. In general, these networks are different for each soloist - they have different time scales (not only for the different musicians but for also for the switching between different ideas) and connectivity to build the sequence of ideas. Thus, this process consists of several attention episodes.

The distributed mind modes that we consider are based on complex brain networks. The neural substrates that underlie spontaneous musical performance have been investigated in (Limb and Braun, 2008). Authors examined improvisation in professional jazz pianists playing on a specially constructed keyboard using fMRI. They found that improvisation was characterized by a dissociated pattern of activity in the prefrontal cortex: deactivation of dorsolateral prefrontal and lateral orbital regions with focal activation of the medial prefrontal cortex. Such a pattern may reflect a combination of psychological processes required for spontaneous improvisation, in which internally motivated, stimulus-independent behaviors develop in the absence of central processes that typically mediate self-monitoring and conscious control of the ongoing performance. To illustrate the main idea about spontaneous chaotic playing with different ideas, we do not take into account in our modeling a very important part of the creativity process - emotion. Usually all partner jazzmen play up to a soloist. We ignored this complication because we believe that it contributes little to our model of creativity while complicating the corresponding dynamical model. Thus, we omit direct 
connections between players, see Figure 16.

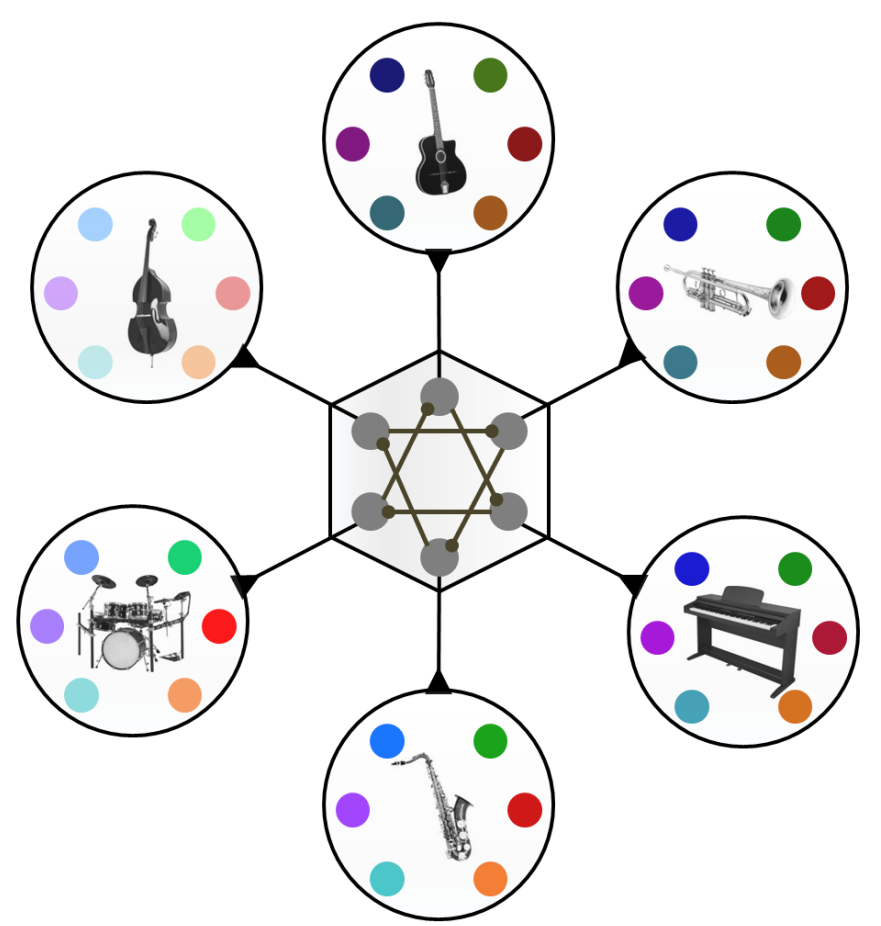

Figure 16 (Color online). Hierarchical attention controls the sequence of the soloists (hexagon network) and soloists control the sequence of creativity ideas (circles).

The corresponding simplified model can be written for $x_{i}^{m}$ depending on an attentional control network similar to (2.3), (2.5) described for $R^{m}(l) \geq 0$ as follows:

$$
\theta^{m} \frac{d R^{m}}{d t}=R^{m} \cdot\left[\gamma^{m}-\sum_{k=1}^{M} \varsigma^{m k} R^{k}\right]
$$

Here, $\theta^{m}$, characterizes the time scale of the switching to $m$-th soloist, $\gamma^{m}$ is the level of excitation of the $m$-th soloist, $\varsigma^{m k} \geq 0$.

The model for the dynamics of the creativity ideas $x_{i}^{m}$ can be written in the form:

$$
\tau_{i}^{m} \frac{d x_{i}^{m}}{d t}=x_{i}^{m} \cdot\left[\sigma_{i}^{m}\left(R^{m}, S^{m}, C^{m}\right)-\sum_{j=1}^{K^{m}} \rho_{i j}^{m} x_{j}^{m}\right]
$$

Here $\tau^{m}$ characterizes the time scale of the ideas switching; $S$ is the vector of sensory inputs and $C$ is the vector of cognitive inputs, $\sigma_{i}^{m}(\ldots) \geq 0 . \rho_{i j}^{m} \geq 0$ is the connection matrix between competitive ideas using by $m$-th soloist. For the simulation of this network, we used the set of parameters described in [P6] (see Appendix 7.2). We specified the functions $\sigma_{i}^{m}$ as $\sigma_{i}^{m}=\sigma_{i_{0}}^{m}\left(S^{m}, C^{m}\right) R^{m}$. The connectivity parameters $\rho_{i j}^{m}$ used to model the 6-mode networks that represent each modality are described in [P2] 
allowing $\pm 2 \%$ variability in these values for each of the networks and finally, the values for $\sigma_{i_{0}}^{m}$ were chosen as in [P4].

The results of the simulation of the dynamics for this model are presented in Figures 17, 18 and 19. Figure 17A represents the time series corresponding to an independent soloist improvisation. Four creativity ideas out of six are plotted for simplicity. Figure 17B corresponds to the same player interacting with the other players as a band. To characterize quantitatively the value of the information generated by the creativity process we calculated the Lyapunov dimension for each network using the Kaplan-Yorke formula:

$$
D_{K Y}=j+\sum_{k=1}^{j} \frac{\lambda_{k}}{\left|\lambda_{j+1}\right|},
$$

where $\lambda_{1} \leq \ldots \leq \lambda_{n}$ are the Lyapunov characteristic exponents and $j$ is the largest integer for which $\lambda_{1}+\ldots+\lambda_{j} \geq 0$. We show the results of the players in the band in the following table (c.f. the value for the individual soloist in the caption of Figure 17A):

\begin{tabular}{|c|c|}
\hline $\begin{array}{c}\text { Network } \\
\text { (soloist \#) }\end{array}$ & $D_{K Y}$ \\
\hline 1 & 5.15 \\
\hline 2 & 5.13 \\
\hline 3 & 4.58 \\
\hline 4 & 5.42 \\
\hline 5 & 6.01 \\
\hline 6 & 5.25 \\
\hline
\end{tabular}
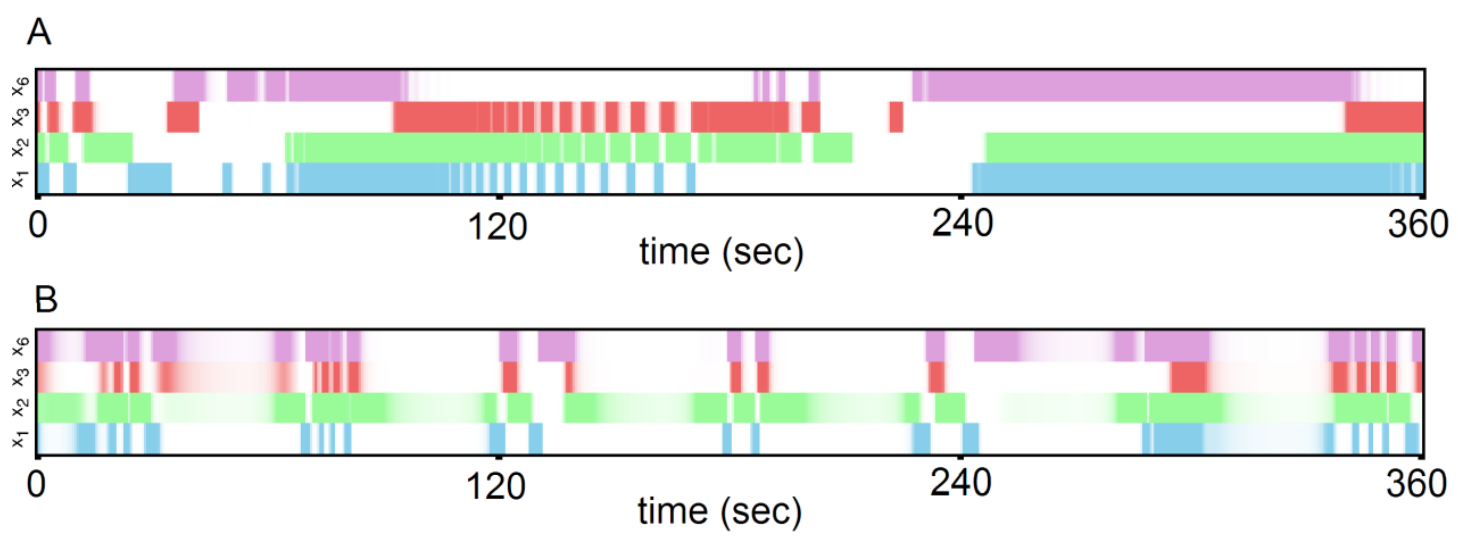

Figure 17 (Color online). Individual soloist improvisation: A - a jazzman independently plays, $D_{K Y}=4.05$. B - the same player activity is sequentially switching with the other players of the band under the attentional network modulation, $D_{K Y}=6.01$ in this case. Different colors represent the temporal patterns corresponding to different creativity tools activated (see Figure 16). 


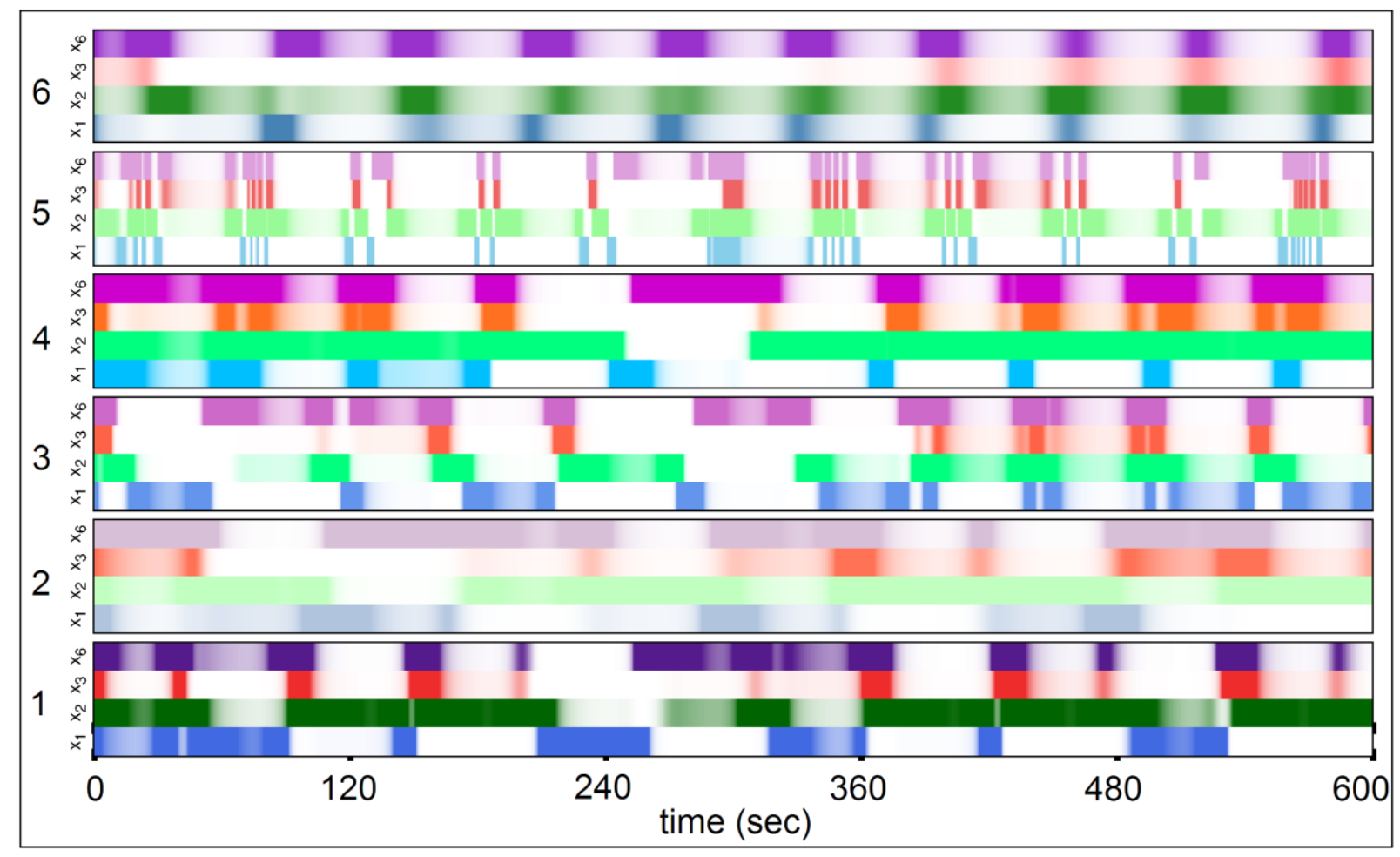

Figure 18 (Color online). Creativity sequential pattern produced by the members of the team (from 1 to 6$)$.

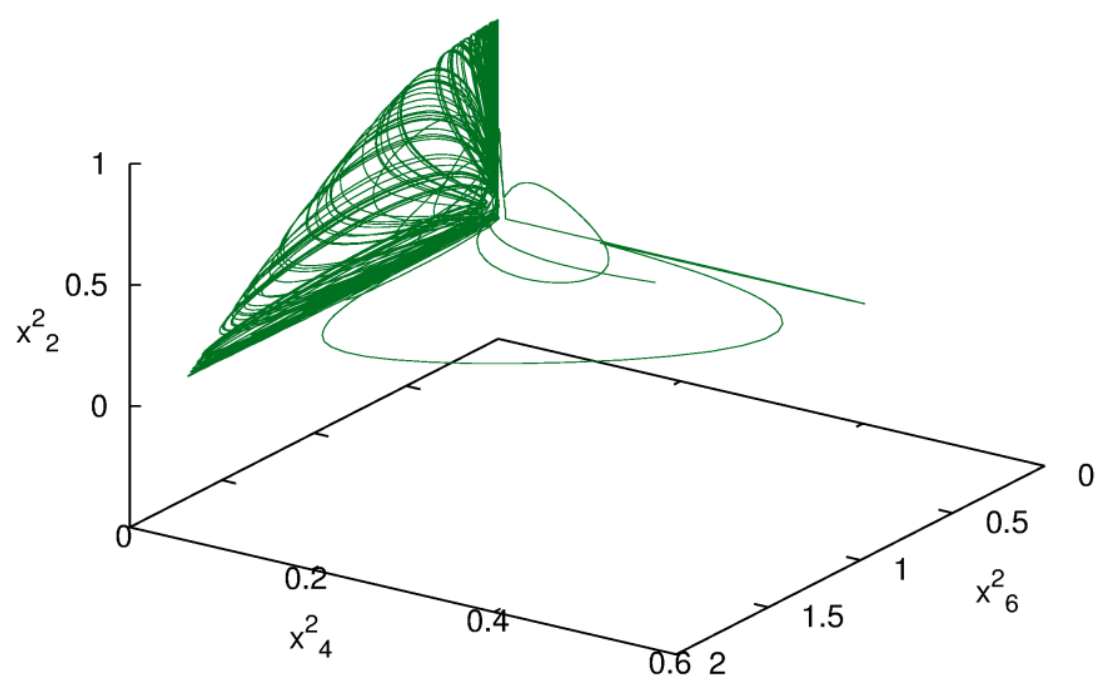

Figure 19 (Color online). 3D projection of the strange attractor of one of the soloist playing in the band. 
Figure 18 shows the switching dynamics of ideas for all six players in the band. Figure 19 shows the chaotic dynamics -strange attractor- for one of the soloist playing in the band. Note that the creativity $D_{K Y}$ is higher when the soloists play as a band. It is important to emphasize that the invention of novel motor sequences in musical improvisation involves choosing a network of brain regions coordinated to generate possible sequences, selecting among them, and executing the decided-upon sequence. The features of these networks form a unique signature of personality and explain the diversity of human creativity ability (Berkowitz and Ansari, 2008; De Manzano and Ullén, 2012; De Manzano et al., 2010; Jerde et al., 2011; Limb and Braun, 2008; Liu et al., 2012).

\subsection{Attention dynamics and creative thinking. Divergence-convergence bifurcation.}

Wallas - as various authors after him - suggested that creative acts run through a divergence-convergence transformation (Wallas, 1926). In a creative process, ideas need to be explored and evaluated as a function of the creative goal, which helps to build useful explorations on alternatives or discard explorations that do not lead to the goal. In these creative processes, even the goal can change as a function of these explorations.

In the framework of such classification, the 'random playing with ideas' that we just discussed above evidently is part of the divergence. What happens next? It is reasonable to suppose that among many ideas or concepts we choose a few of them - A, $\mathrm{B}$ and $\mathrm{C}$, which are the closest to our cognitive goal. At the divergence stage, our attention is shared among those concepts. For some time interval, this is a static stage. Afterwards, the role of attention dynamics increases and we can see the sequential switching between A, B and C (see Figure 20).

Our general model of attention dynamics (see equations (5.1), (5.2) above) is able to describe the transition between convergence and divergence as bifurcations that are controlled by 'evaluating intrinsic stimuli' which is an estimator of the distance between the goal of the creativity process and members of a 'pool of ideas'. This bifurcation is presented in Figure 20. 


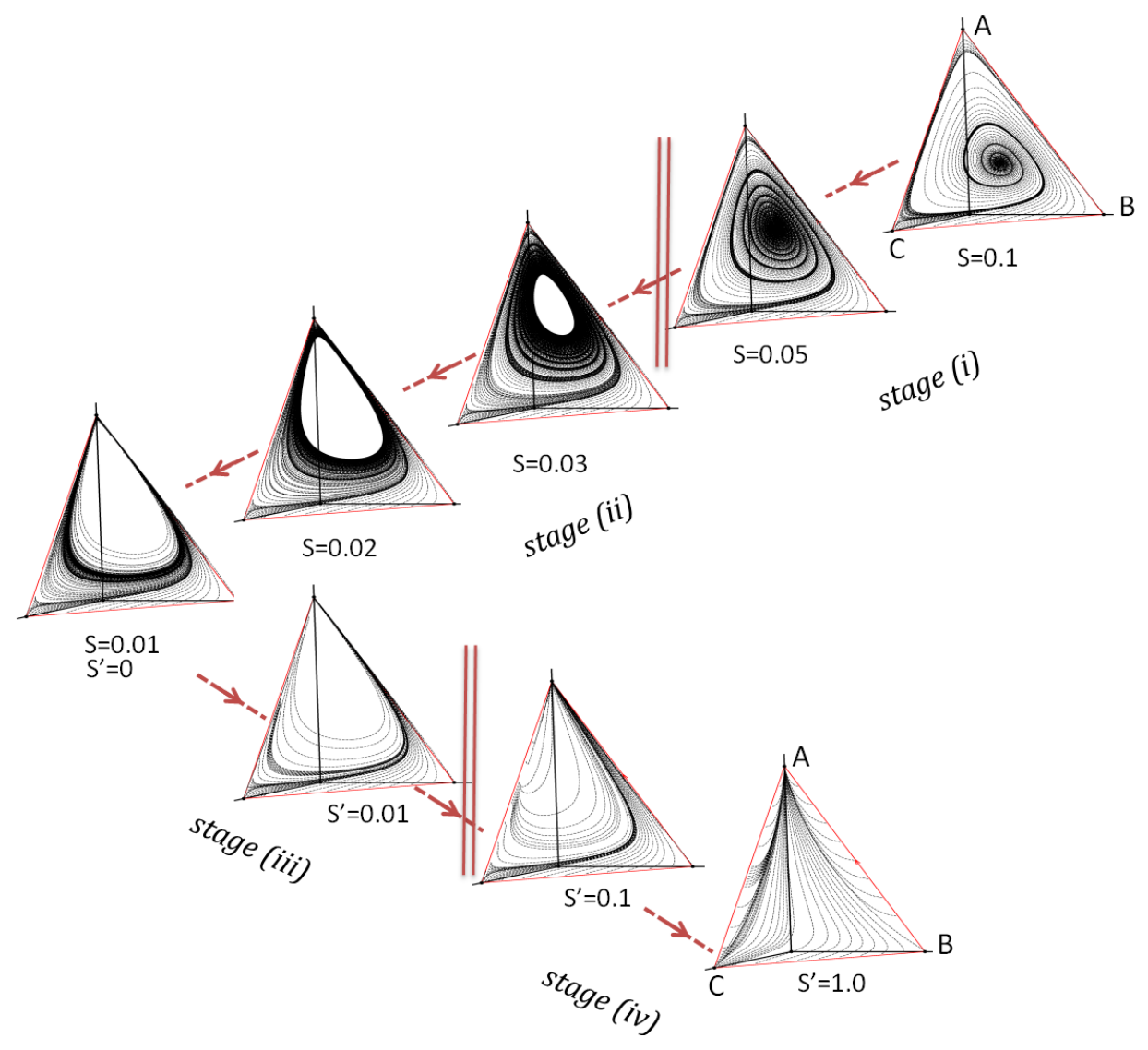

Figure 20 (Color online). Transitions between different stages of creativity acts: divergence and convergence. Creativity stage (i) is represented in the phase space of the system (5.1) by a stable fixed point - attention is distributed among divergent ideas that weakly interact with each other; stage (ii) is represented by a stable limit cycle that emerges when the distance between the goal and ideas A, B and C becomes small (parameter $S \rightarrow 0$ ); on stage (iii) the idea A becomes prevalent (the parameter $S^{\prime}$ that characterizes the distance of ideas B and $\mathrm{C}$ to the goal increases); and finally on the stage (iv) the system arrives to a final decision - A. The parameters used to model the attention dynamics between ideas A, B and C are described by [P7] in the appendix with $S=0.01,0.02,0.03,0.05,0.1, S^{\prime}=0$ for stages (i) and (ii). The same parameters with $S=0.01, S^{\prime}=0.00,0.01,0.10,1.00$ are used for stages (iii) and (iv).

Thus we have considered the possible dynamical mechanisms involved in decisionmaking among three alternatives. The goal-relevant alternative A is supported by the 'minimal distance to the goal representation' and competes with the choice alternatives $B$ and $C$ through mutual inhibition supported by increasing distances to the goal of alternatives B and C (parameter $S^{\prime}$ increases). Creativity requires that we first focus our attention on something even for a short time - typically something that we have not focused much attention on before and that escaped our current thinking. Evidently, in the general case, the model has to include sufficient modalities in order to be able to 
generate new creative ideas and reject thoughts that seem not productive or too ridiculous to participate in the creativity process.

\section{Conclusions}

Perception and attention are interrelated hierarchical dynamical processes that depend on both sensory cues and memory that represents previously learned information about sequential events and cognitive tasks and plans (Botvinick, 2008; Zacks et al., 2007). To build an operational model of attention dynamics, we suggested in this paper the following logical chain of steps: a) to process the spatiotemporal imaging data in order to extract the projection functions which make a bridge between the spatial and temporal activity of specific sequential cognitive modes; b) to find the basic principles for the formulation of a canonic low-dimensional dynamical model that can be a building block in the hierarchical construction of different cognitive episodes; c) to provide in this canonic model and its generalization control parameters for modeling different cognitive tasks and behavioral plans based on the knowledge of the hierarchical organization that corresponds to specific functional brain networks; and finally, d) to choose the architecture of the hierarchical cognitive model in order to guaranty the robustness against noise and, at the same time, the sensitivity to the changing environment and the intrinsic state of the actor.

In this paper we have demonstrated the power of nonlinear dynamics methods on the steps b) and, partially, d). To complete the step d) it is necessary to understand in more detail the functional reorganization of brain networks on the different attentional (cognitive) sequential episodes involved in the performance of a composite cognitive goal. For example, to send a message with our cell phone we need to turn on the cell phone, to find the recipient, to type the message, and finally to send the message. Different brain networks are responsible for the execution of these subtasks. Thus, we have to understand the mechanisms of attention switching from one stage to next one keeping the continuity of the main goal performance.

In relation to step a), modeling approaches have to be developed hand in hand with novel experimental protocols that take into account the transient nature of brain dynamics. Novel experiments on sequential brain activity using online model-controlled neurofeedback could contribute to progress in this direction. 


\section{Appendices}

\subsection{Robustness of sequential cognitive dynamics based on heteroclinic sequences}

To analyze the robustness of the heteroclinic sequence against small perturbations we consider here the case where metastable states are just saddle equilibrium points $S_{1}, \ldots, S_{N}$ of a system $\dot{x}=F(x)$, where $x$ is $n$-dimensional vector (Afraimovich et al., 2012). Let the eigenvalues $\lambda_{1}^{(i)}, \ldots, \lambda_{n}^{(i)}$ of the matrix of the system linearized at $S_{i}$ be ordered in such a way that $\lambda_{1}^{(i)}>\ldots \geq \operatorname{Re} \lambda_{m_{i}}^{(i)}>0>\operatorname{Re} \lambda_{m_{i}+1}^{(i)} \geq \ldots \geq \operatorname{Re} \lambda_{n}^{(i)}$. Then, on the $m_{i}$ -dimensional unstable manifold $W_{i}^{u}$ there is a strongly unstable one-dimensional manifold $W_{i}^{u u}$ tangent to the eigenvector corresponding to $\lambda_{1}^{(i)}$. It consists of $S_{i}$ and two trajectories $\Gamma^{+}$and $\Gamma^{-}$, i.e. $W_{i}^{u u}=\Gamma_{i}^{+} \cup S_{i} \cup \Gamma_{i}^{-}$. If $\Gamma_{i}^{+} \subset W_{i+1}^{s}, i=1, \ldots, N-1$, the set $\Gamma:=\bigcup_{i=1}^{N} S_{i} \bigcup_{i=1}^{N-1} \Gamma_{i}^{+}$is said to be a heteroclinic sequence. If $S_{N} \equiv S_{1}$ then $\Gamma$ is called the heteroclinic cycle (contour). Heteroclinic cycles can serve as attractors provided that the saddle values $-\frac{\operatorname{Re} \lambda_{m_{i}+1}^{(i)}}{\lambda_{1}^{(i)}}=v_{i}>1$ and $\Gamma$ is characterized by $\operatorname{dim} W_{i}^{u}=1, i=1, \ldots, N$. Such a situation is realized in systems for which the winnerless competition dynamics takes place (Rabinovich et al., 2006c). If $S_{N} \neq S_{1}$ then one deals with a heteroclinic sequence. If $v_{i}>1, i=1, \ldots, N$, and $\operatorname{dim} W_{i}^{u}=1$ then still some kind of stability holds: any orbit going through an initial point in a vicinity of $S_{1}$, stays in a neighborhood of $\Gamma$ until it reaches a neighborhood of $S_{N}$. If $\operatorname{dim} W_{i}^{u}>1$ for some $i$ then most such orbits still do not leave a neighborhood of $\Gamma$ but some of them ( $\cong 20 \%$ if $\operatorname{dim} W_{i}^{u}=2$ (Afraimovich et al., 2013)) leave a vicinity of $\Gamma$ and go to other attracting sets in the phase space. The temporal characteristics of the transients are related to the exit problem for small random perturbations of dynamical systems with saddle sets. A local stability analysis in the vicinity of a saddle fixed point allows to estimate the characteristic time that the system spends in the vicinity of the saddle as $\tau(p)=1 / \lambda_{1}(i) \ln (1 /|\eta|)$, where $\tau(p)$ is the mean passage time, $|\eta|$ is the level of noise, and $\lambda_{1}{ }^{(i)}$ is the maximum eigenvalue corresponding to the unstable separatrices of the saddle. There exist some results about heteroclinic channel stability where metastable sets are represented by saddle limit cycles (Komarov et al., 2009).

Intuitively it is clear that the heteroclinic channel has to be robust against small perturbations if all metastable states that form the heteroclinic chain have saddle values larger than one, i.e., thecompression of the phase volume in the vicinity of the sequence stronger then stretching - the trajectories that come in a vicinity of a saddle sequence become prisoners of the channel.- A rigorous analysis of the structural stability of the heteroclinic channel supports this intuition, see (M. I. Rabinovich et al., 
2008).

\subsection{Simulation parameters}

Sect 3.3

[P1] $M=2, K^{m}=6, \quad m=1,2 ; \tau_{i}^{1}=1.00, \tau_{i}^{2}=0.90$,

[P2] Parameters $\rho_{i j}^{m}$ used to model the 6-mode networks that represent each modality for $\quad m=1 \quad$ are $\quad \rho_{13}^{1}=\rho_{35}^{1}=\rho_{51}^{1}=5 ; \quad \rho_{46}^{1}=\rho_{24}^{1}=\rho_{62}^{1}=2$; $\rho_{16}^{1}=\rho_{21}^{1}=\rho_{32}^{1}=\rho_{43}^{1}=\rho_{54}^{1}=\rho_{65}^{1}=1.5 ; \rho_{11}^{m}=\rho_{22}^{m}=\rho_{33}^{m}=\rho_{44}^{m}=\rho_{55}^{m}=\rho_{66}^{m}=1$.

[P3] Parameters $\rho_{i j}^{m}$ used to model the 6-mode networks that represent each modality for $\quad m=2 \quad$ are $\quad \rho_{13}^{2}=\rho_{35}^{2}=\rho_{51}^{2}=5.02 ; \quad \rho_{46}^{2}=\rho_{24}^{2}=\rho_{62}^{2}=2.02$; $\rho_{16}^{2}=\rho_{21}^{2}=\rho_{32}^{2}=\rho_{43}^{2}=\rho_{54}^{2}=\rho_{65}^{2}=1.52 ; \rho_{11}^{m}=\rho_{22}^{m}=\rho_{33}^{m}=\rho_{44}^{m}=\rho_{55}^{m}=\rho_{66}^{m}=1$.

[P4] Values for $\sigma_{i_{0}}^{m}$ were chosen as $\sigma_{1_{0}}^{m}=1.730, \sigma_{2_{0}}^{m}=1.123, \sigma_{3_{0}}^{m}=1.301, \sigma_{4_{0}}^{m}=1.203$, $\sigma_{5_{0}}^{m}=1.458, \sigma_{6_{0}}^{m}=1.903$.

\section{Sect 4.1}

[P5] The parameters $\varsigma^{m k}$ used to model the attention dynamics between modalities are $\varsigma^{11}=\varsigma^{22}=\varsigma^{33}=1, \quad \varsigma^{23}=\gamma^{2} / \gamma^{3}-0.10, \quad \varsigma^{32}=\gamma^{3} / \gamma^{2}-0.12, \quad \varsigma^{12}=\varsigma_{0}^{12}+S^{\prime} \sin (\omega \tau+3 / 29)$, $\varsigma^{13}=\varsigma_{0}^{13}+S^{\prime} \sin (\omega \tau+21 / 5), \quad \varsigma^{21}=\varsigma_{0}^{21}+S^{\prime} \sin (\omega \tau+13 / 3), \quad \varsigma^{31}=\varsigma_{0}^{31}+S^{\prime} \sin (\omega \tau+7 / 11)$, $\varsigma_{0}^{12}=\gamma^{1} / \gamma^{2}+0.12, \quad \varsigma_{0}^{13}=\gamma^{1} / \gamma^{3}+0.10, \quad \varsigma_{0}^{21}=\gamma^{2} / \gamma^{1}+0.01, \quad \varsigma_{0}^{31}=\gamma^{3} / \gamma^{1}-0.01$, where $\gamma^{1}=1.5, \gamma^{2}=1.426, \gamma^{3}=0.956, S^{\prime}=0.15$ and $\omega=4 \pi / 3$.

\section{Sect 5.4}

[P6] $M=6, \theta^{m}=1, \tau_{1}^{m}=0.37, \tau_{2}^{m}=0.76, \tau_{3}^{m}=0.16, \tau_{4}^{m}=0.42, \tau_{5}^{m}=0.05, \tau_{6}^{m}=0.85$, $K^{m}=6, m=1, \ldots, 6$. The parameters $\varsigma^{m k}$ used to model the attentional modes are $\varsigma^{13}=\varsigma^{35}=\varsigma^{51}=5 ; \quad \varsigma^{46}=\varsigma^{24}=\varsigma^{62}=3 ; \quad \varsigma^{11}=\varsigma^{22}=\varsigma^{33}=\varsigma^{44}=\varsigma^{55}=\varsigma^{66}=1$. The values of $\gamma^{m}$ were chosen in these specific simulations as $\gamma^{1}=1.650, \gamma^{2}=1.356, \gamma^{3}=1.473$, $\gamma^{4}=1.781, \gamma^{5}=1.804, \gamma^{6}=1.269$.

\section{Sect 5.5}

[P7] The parameters $\varsigma^{m k}$ are $\varsigma^{11}=\varsigma^{22}=\varsigma^{33}=1, \varsigma^{12}=\frac{\gamma^{1}}{\gamma^{2}}+0.12, \quad \varsigma^{32}=\frac{\gamma^{3}}{\gamma^{2}}-0.12$, $\varsigma^{13}=\frac{\gamma^{1}}{\gamma^{3}}+0.10, \quad \varsigma^{23}=\frac{\gamma^{2}}{\gamma^{3}}-0.10, \quad \varsigma^{21}=\frac{\gamma^{2}}{\gamma^{1}}+S+S^{\prime}, \quad \varsigma^{31}=\frac{\gamma^{3}}{\gamma^{1}}-S+S^{\prime} ; \quad$ and $\quad \gamma^{1}=1.5$, $\gamma^{2}=1.426$ and $\gamma^{3}=0.956$ 


\section{Acknowledgments}

Mikhail I. Rabinovich was supported by ONR Grant N00014310205, Irma Tristan was supported by UC MEXUS-CONACYT Postdoctoral Fellowship and Pablo Varona was supported by MINECO TIN2012-30883 and UAM CEMU 2012-004. We thank Dr. David Pintsov for his help to edit the manuscript.

\section{References}

Afraimovich, V., Rabinovich, M., Varona, P., 2012. Short Guide to Modern Nonlinear Dynamics, in: Rabinovich, M.I., Friston, K., Varona, P. (Eds.), Principles of Brain Dynamics: Global State Interactions. MIT Press, Cambridge, MA, p. 313.

Afraimovich, V., Tristan, I., Varona, P., Rabinovich, M., 2013. Transient Dynamics in Complex Systems: Heteroclinic Sequences with Multidimensional Unstable Manifolds. Discontinuity, Nonlinearity, Complex. 2, 21-41. doi:10.5890/DNC.2012.11.001

Ahn, A.H., 2012. Southern Headache Society supplement: the neurobiology of throbbing pain in migraine. Headache 52 Suppl 1, 12-4. doi:10.1111/j.15264610.2012.02136.x

Alvarez, G.A., Cavanagh, P., 2004. The capacity of visual short-term memory is set both by visual information load and by number of objects. Psychol. Sci. 15, 106-11. doi:10.1111/j.0963-7214.2004.01502006.x

Anderson, J.S., Ferguson, M.A., Lopez-Larson, M., Yurgelun-Todd, D., 2010. Topographic maps of multisensory attention. Proc. Natl. Acad. Sci. U. S. A. 107, 20110-20114.

Ansersson, D.E., Andersson, A.E., C., M., 2013. Handbook of Creative Cities. Edward Elgar Pub.

Baddeley, A., 1992. Working memory. Science (80-. )., New Series 255, 556-559.

Banerjee, A., Pillai, A.S., Horwitz, B., 2012. Using large-scale neural models to interpret connectivity measures of cortico-cortical dynamics at millisecond temporal resolution. Front. Syst. Neurosci. 5, 102. doi:10.3389/fnsys.201

Barkley, R.A., 1997. Behavioral inhibition, sustained attention, and executive functions: constructing a unifying theory of ADHD. Psychol. Bull. 121, 65-94.

Bell, A.J., Sejnowski, T.J., 1995. An information-maximisation approach to blind separation and blind deconvolution. Neural Comput. 7, 1129-1159. doi:10.1162/neco.1995.7.6.1129

Ben-Simon, E., Podlipsky, I., Okon-Singer, H., Gruberger, M., Cvetkovic, D., Intrator, N., Hendler, T., 2013. The dark side of the alpha rhythm: fMRI evidence for induced alpha modulation during complete darkness. Eur. J. Neurosci. 37, 795-803.

Bergson, H., 1920. Mind-energy: lectures and essays. H. Holt, New York.

Berkowitz, A.L., Ansari, D., 2008. Generation of novel motor sequences: the neural correlates of musical improvisation. Neuroimage 41, 535-543. 
Bick, C., Rabinovich, M.I., 2009. Dynamical origin of the effective storage capacity in the brain's working memory. Phys. Rev. Lett. 103, 218101.

doi:10.1103/PhysRevLett.103.218101

Bingel, U., Rose, M., Gläscher, J., Büchel, C., 2007. fMRI reveals how pain modulates visual object processing in the ventral visual stream. Neuron 55, 157-167.

Bonsall, M.B., Wallace-Hadrill, S.M.A., Geddes, J.R., Goodwin, G.M., Holmes, E.A., 2012. Nonlinear time-series approaches in characterizing mood stability and mood instability in bipolar disorder. Proc. Biol. Sci. 279, 916-24. doi:10.1098/rspb.2011.1246

Botvinick, M.M., 2008. Hierarchical models of behavior and prefrontal function. Trends Cogn. Sci. 12, 201-208.

Bressler, S.L., Menon, V., 2010. Large-scale brain networks in cognition: emerging methods and principles. Trends Cogn. Sci. 14, 277-290.

Buschman, T.J., Miller, E.K., 2010. Shifting the Spotlight of Attention: Evidence for Discrete Computations in Cognition. Front. Hum. Neurosci. 4, 9.

Bystritsky, A., Nierenberg, A.A., Feusner, J.D., Rabinovich, M., 2012. Computational nonlinear dynamical psychiatry: a new methodological paradigm for diagnosis and course of illness. J. Psychiatr. Res. 46, 428-35.

doi:10.1016/j.jpsychires.2011.10.013

Chan, S.C.C., Chan, C.C.H., Kwan, A.S.K., Ting, K.-H., Chui, T.-Y., 2012. Orienting Attention Modulates Pain Perception: An ERP Study. PLoS One 7, e40215. doi:10.1371/journal.pone.0040215

Ciesielski, K.T., Harris, R.J., 1997. Factors related to performance failure on executive tasks in autism. Child Neuropsychol. 3, 1-12.

Clayton, I.C., Richards, J.C., Edwards, C.J., 1999. Selective attention in obsessivecompulsive disorder. J. Abnorm. Psychol. 108, 171-175.

Corbetta, M., Patel, G., Shulman, G.L., 2008. The Reorienting System of the Human Brain: From Environment to Theory of Mind. Neuron 58, 306-324.

Cowell, R.A., Bussey, T.J., Saksida, L.M., 2012. Empiricists are from Venus, modelers are from Mars: Reconciling experimental and computational approaches in cognitive neuroscience. Neurosci. Biobehav. Rev. doi:10.1016/j.neubiorev.2012.08.008

Dagenbach, D., Carr, T.H. (Eds.), 1994. Inhibitory processes in attention, memory, and language, 1994th ed. Academic Press Inc.

De Freitas, J., Liverence, B.M., Scholl, B., 2012. Attentional rhythm: A temporal analogue of object-based attention. J. Vis. 12, 257-257. doi:10.1167/12.9.257

De Manzano, Ö., Cervenka, S., Karabanov, A., Farde, L., Ullén, F., 2010. Thinking Outside a Less Intact Box: Thalamic Dopamine D2 Receptor Densities Are Negatively Related to Psychometric Creativity in Healthy Individuals. PLoS One 5, 6.

De Manzano, O., Theorell, T., Harmat, L., Ullén, F., 2010. The psychophysiology of flow during piano playing. Emotion 10, 301-311.

De Manzano, Ö., Ullén, F., 2012. Goal-independent mechanisms for free response generation: creative and pseudo-random performance share neural substrates. Neuroimage 59, 772-80. doi:10.1016/j.neuroimage.2011.07.016

De Vico Fallani, F., Nicosia, V., Sinatra, R., Astolfi, L., Cincotti, F., Mattia, D., Wilke, C., Doud, A., Latora, V., He, B., Babiloni, F., 2010. Defecting or not defecting: How to 
"read" human behavior during cooperative games by EEG measurements. PLoS One 5.

Deco, G., Jirsa, V., McIntosh, A.R., Sporns, O., Kötter, R., 2009. Key role of coupling, delay, and noise in resting brain fluctuations. Proc. Natl. Acad. Sci. U. S. A. 106, 1030210307.

Deco, G., Jirsa, V.K., 2012. Ongoing Cortical Activity at Rest: Criticality, Multistability, and Ghost Attractors. J. Neurosci. doi:10.1523/JNEUROSCI.2523-11.2012

Deco, G., Jirsa, V.K., McIntosh, A.R., 2011. Emerging concepts for the dynamical organization of resting-state activity in the brain. Nat. Rev. Neurosci. 12, 43-56.

Deco, G., Jirsa, V.K., McIntosh, A.R., 2013. Resting brains never rest: computational insights into potential cognitive architectures. Trends Neurosci. 36, 268-74.

Deco, G., Jirsa, V.K., Robinson, P.A., Breakspear, M., Friston, K., 2008. The dynamic brain: From spiking neurons to neural masses and cortical fields. PLoS Comput. Biol. 4, e1000092. doi:10.1371/journal.pcbi.1000092

Deco, G., Rolls, E.T., 2003. Attention and working memory: a dynamical model of neuronal activity in the prefrontal cortex. Eur. J. Neurosci. 18, 2374-2390.

Deco, G., Rolls, E.T., 2005. Attention, short-term memory, and action selection: a unifying theory. Prog. Neurobiol. 76, 236-56. doi:10.1016/j.pneurobio.2005.08.004

Dempster, F.N., Brainerd, C.J., 1995. Interference and inhibition in cognition, and inhibition in cognition. Academic Press.

Desimone, R., Duncan, J., 1995. Neural mechanisms of selective visual attention. Annu. Rev. Neurosci. 18, 193-222. doi:10.1146/annurev.ne.18.030195.001205

Dieter, K.C., Tadin, D., 2011. Understanding attentional modulation of binocular rivalry: a framework based on biased competition. Front. Hum. Neurosci. 5, 155. doi:10.3389/fnhum.2011.00155

Dumas, G., Lachat, F., Martinerie, J., Nadel, J., George, N., 2011. From social behaviour to brain synchronization: Review and perspectives in hyperscanning. IRBM 32, 4853.

Dumas, G., Nadel, J., Soussignan, R., Martinerie, J., Garnero, L., 2010. Inter-brain synchronization during social interaction. PLoS One 5.

Duncan, J., 2013. The structure of cognition: attentional episodes in mind and brain. Neuron 80, 35-50. doi:10.1016/j.neuron.2013.09.015

Eccleston, C., Crombez, G., 1999. Pain demands attention: A cognitive-affective model of the interruptive function of pain. Psychol. Bull. 125, 356-366. doi:10.1037/00332909.125.3.356

Edin, F., Klingberg, T., Johansson, P., McNab, F., Tegnér, J., Compte, A., 2009. Mechanism for top-down control of working memory capacity. Proc Natl Acad Sci U S A 106, 6802-6807. doi:10.1073/pnas.0901894106

Feldman, H., Friston, K.J., 2010. Attention, uncertainty, and free-energy. Front. Hum. Neurosci. 4, 215. doi:10.3389/fnhum.2010.00215

Friston, K.J., Frith, C.D., Frackowiak, R.S., 1993. Principal Component Analysis Learning Algorithms - A Neurobiological Analysis. Proc. R. Soc. B Biol. Sci. 254, 47-54.

Gaddes, W.H., Edgell, D., 1994. Learning Disabilities and Brain Function: A Neuropsychological Approach. Springer-Verlag, New York. 
García-Blanco, A.C., Perea, M., Livianos, L., 2013. Mood-congruent bias and attention shifts in the different episodes of bipolar disorder. Cogn. Emot. 1-8. doi:10.1080/02699931.2013.764281

George, J.M., 2000. Emotions and leadership: The role of emotional intelligence. Hum. Relations 53, 1027-1055 ST - Emotions and leadership : The role.

Ghosh, A., Rho, Y., McIntosh, A.R., Kötter, R., Jirsa, V.K., 2008. Noise during rest enables the exploration of the brain's dynamic repertoire. PLoS Comput. Biol. 4, e1000196.

Goadsby, P.J., 2013. All that is obvious is not clear: what is the origin of throbbing pain in migraine? Pain 154, 971-970. doi:10.1016/j.pain.2013.04.019

Goldman-Rakic, P.S., 1988. Topography of cognition: parallel distributed networks in primate association cortex. Annu. Rev. Neurosci. 11, 137-56. doi:10.1146/annurev.ne.11.030188.001033

Gottlieb, J., Balan, P., 2010. Attention as a decision in information space. Trends Cogn. Sci. 14, 240-8. doi:10.1016/j.tics.2010.03.001

Hari, R., Salmelin, R., 1997. Human cortical oscillations: A neuromagnetic view through the skull. Trends Neurosci.

Hasenkamp, W., Wilson-Mendenhall, C.D., Duncan, E., Barsalou, L.W., 2012. Mind wandering and attention during focused meditation: A fine-grained temporal analysis of fluctuating cognitive states. Neuroimage $59,750-760$. doi:10.1016/j.neuroimage.2011.07.008

Hasson, U., Yang, E., Vallines, I., Heeger, D.J., Rubin, N., 2008. A hierarchy of temporal receptive windows in human cortex. J. Neurosci. 28, 2539-2550. doi:10.1523/JNEUROSCI.5487-07.2008

Hermundstad, A.M., Bassett, D.S., Brown, K.S., Aminoff, E.M., Clewett, D., Freeman, S., Frithsen, A., Johnson, A., Tipper, C.M., Miller, M.B., Grafton, S.T., Carlson, J.M., 2013. Structural foundations of resting-state and task-based functional connectivity in the human brain. Proc. Natl. Acad. Sci. U. S. A. 110, 6169-74. doi:10.1073/pnas.1219562110

Hsieh, L.-T., Ekstrom, A.D., Ranganath, C., 2011. Neural oscillations associated with item and temporal order maintenance in working memory. J. Neurosci. 31, 1080310810.

Huerta, R., Rabinovich, M., 2004. Reproducible sequence generation in random neural ensembles. Phys Rev Lett 93, 238104.

Jerde, T.A., Childs, S.K., Handy, S.T., Nagode, J.C., Pardo, J. V, 2011. Dissociable systems of working memory for rhythm and melody. Neuroimage 57, 1572-1579.

Johnson, S., 2012. Select Essays of Dr. Johnson, Volume 2. Nabu Press.

Jones, L.M., Fontanini, A., Sadacca, B.F., Miller, P., Katz, D.B., 2007. Natural stimuli evoke dynamic sequences of states in sensory cortical ensembles. Proc Natl Acad Sci USA 104, 18772-18777. doi:10.1073/pnas.0705546104

Kahneman, D. (Ed.), 1973. Attention and Effort. Englewood Cliffs, N.J. Prentice Hall.

Kelso, J.A., Fuchs, A., Lancaster, R., Holroyd, T., Cheyne, D., Weinberg, H., 1998. Dynamic cortical activity in the human brain reveals motor equivalence. Nature 392, 814-8.

Kiebel, S.J., Daunizeau, J., Friston, K.J., 2008. A hierarchy of time-scales and the brain. PLoS Comput Biol 4, e1000209. doi:10.1371/journal.pcbi.1000209

Koch, C., Tsuchiya, N., 2007. Attention and consciousness: two distinct brain processes. Trends Cogn. Sci. 11, 16-22. 
Komarov, M.A., Osipov, G. V., Suykens, J.A.K., 2009. Sequentially activated groups in neural networks. Europhys. Lett. 86, 60006. doi:10.1209/0295-5075/86/60006

Konvalinka, I., Roepstorff, A., 2012. The two-brain approach: how can mutually interacting brains teach us something about social interaction? Front. Hum. Neurosci. 6, 215.

Kurikawa, T., Kaneko, K., 2013. Embedding Responses in Spontaneous Neural Activity Shaped through Sequential Learning. PLoS Comput. Biol. 9, e1002943. doi:10.1371/journal.pcbi.1002943

Lamme, V.A.F., 2003. Why visual attention and awareness are different. Trends Cogn. Sci. 7, 12-18. doi:10.1016/S1364-6613(02)00013-X

Lappe, C., Steinsträter, O., Pantev, C., 2013. Rhythmic and melodic deviations in musical sequences recruit different cortical areas for mismatch detection. Front. Hum. Neurosci. 7, 260. doi:10.3389/fnhum.2013.00260

Lee, K., 2008. Guiding attention by cooperative cues. J. Comput. Sci. Technol. 23, 874884.

Lee, U., Mashour, G.A., Kim, S., Noh, G.-J., Choi, B.-M., 2009. Propofol induction reduces the capacity for neural information integration: implications for the mechanism of consciousness and general anesthesia. Conscious. Cogn. 18, 56-64.

Lewis-Peacock, J.A., Drysdale, A.T., Oberauer, K., Postle, B.R., 2012. Neural evidence for a distinction between short-term memory and the focus of attention. J. Cogn.

Neurosci. 24, 61-79. doi:10.1162/jocn_a_00140

Limb, C.J., Braun, A.R., 2008. Neural substrates of spontaneous musical performance: an FMRI study of jazz improvisation. PLoS One 3, e1679. doi:10.1371/journal.pone.0001679

Lindenberger, U., Li, S.-C., Gruber, W., Müller, V., 2009. Brains swinging in concert: cortical phase synchronization while playing guitar. BMC Neurosci. 10, 22.

Liu, S., Chow, H.M., Xu, Y., Erkkinen, M.G., Swett, K.E., Eagle, M.W., Rizik-Baer, D.A., Braun, A.R., 2012. Neural correlates of lyrical improvisation: an FMRI study of freestyle rap. Sci. Rep. 2, 834. doi:10.1038/srep00834

Liverence, B.M., Scholl, B.J., 2012. Discrete events as units of perceived time. J. Exp. Psychol. Hum. Percept. Perform. 38, 549-54. doi:10.1037/a0027228

Macleod, C.M., Dodd, M.D., Sheard, E.D., Wilson, D.E., Bibi, U., 2003. In Opposition to Inhibition. Life Sci. 7439, 163-214. doi:10.1016/S0079-7421(03)01014-4

Makeig, S., Gramann, K., Jung, T.-P., Sejnowski, T.J., Poizner, H., 2009. Linking brain, mind and behavior. Int. J. Psychophysiol. 73, 95-100.

Makeig, S., Jung, T.-P., Bell, A.J., Ghahremani, D., Sejnowski, T.J., 1997. Blind separation of auditory event-related brain responses into independent components. Proc. Natl. Acad. Sci. U. S. A. 94, 10979-10984.

Mao, W., Wang, Y., 2008. The active inhibition for the processing of visual irrelevant conflict information. Int. J. Psychophysiol. 67, 47-53.

Marois, R., Ivanoff, J., 2005. Capacity limits of information processing in the brain. Trends Cogn. Sci. 9, 296-305. doi:10.1016/j.tics.2005.04.010

Mazor, O., Laurent, G., 2005. Transient dynamics versus fixed points in odor representations by locust antennal lobe projection neurons. Neuron 48, 661-673. doi:10.1016/j.neuron.2005.09.032 
McIntosh, A.R., Bookstein, F.L., Haxby, J. V, Grady, C.L., 1996. Spatial pattern analysis of functional brain images using partial least squares. Neuroimage 3, 143-57. doi:10.1006/nimg.1996.0016

Meehan, T.P., Bressler, S.L., 2012. Neurocognitive networks: Findings, models, and theory. Neurosci. Biobehav. Rev. 36, 2232-2247. doi:10.1016/j.neubiorev.2012.08.002

Mesulam, M., 1998. From sensation to cognition. Brain 121, 1013-1052. doi:10.1093/brain/121.6.1013

Mirza, A.F., Mo, J., Holt, J.L., Kairalla, J.A., Heft, M.W., Ding, M., Ahn, A.H., 2012. Is There a Relationship between Throbbing Pain and Arterial Pulsations? J. Neurosci. 32, 7572-6. doi:10.1523/JNEUROSCI.0193-12.2012

Muller, J., Roberts, J.E., 2005. Memory and attention in Obsessive-Compulsive Disorder: a review. J. Anxiety Disord. 19, 1-28.

Müller, V., Sänger, J., Lindenberger, U., 2013. Intra- and Inter-Brain Synchronization during Musical Improvisation on the Guitar. PLoS One 8.

Murphy, F.C., Sahakian, B.J., 2001. Neuropsychology of bipolar disorder. Br. J. Psychiatry 178, s120-s127. doi:10.1192/bjp.178.41.s120

Nestor, P., Klein, K., Pomplun, M., Niznikiewicz, M., McCarley, R., 2010. Gaze cueing of attention in schizophrenia: individual differences in neuropsychological functioning and symptoms. J. Clin. Exp. Neuropsychol. 32, 281-288. doi:10.1080/13803390902984472

Nigg, J.T., 2001. Is ADHD a disinhibitory disorder? Psychol. Bull. 127, 571-598. doi:10.1037/0033-2909.127.5.571

Nobre, A.C., Stokes, M.G., 2011. Attention and short-term memory: crossroads. Neuropsychologia 49, 1391-2. doi:10.1016/j.neuropsychologia.2011.04.014

Norman, D.A., Bobrow, D.G., 1975. On data-limited and resource-limited processes. Cogn. Psychol. 7, 44-64. doi:10.1016/0010-0285(75)90004-3

Nowotny, T., Rabinovich, M.I., 2007. Dynamical origin of independent spiking and bursting activity in neural microcircuits. Phys. Rev. Lett. 98, 128106.

Oberauer, K., Kliegl, R., 2006. A formal model of capacity limits in working memory. J. Mem. Lang. 55, 601-626. doi:DOI: 10.1016/j.jml.2006.08.009

Park, H.-J., Friston, K., 2013. Structural and functional brain networks: from connections to cognition. Science (80-. ). 342, 1238411. doi:10.1126/science.1238411

Pascanu, R., Jaeger, H., 2011. A neurodynamical model for working memory. Neural Netw 24, 199-207. doi:10.1016/j.neunet.2010.10.003

Pearson, K., 1901. On lines and planes of closest fit to systems of points in space. Philos. Mag., 6 2, 559-572. doi:10.1080/14786440109462720

Pereira, F., Mitchell, T., Botvinick, M., 2009. Machine learning classifiers and fMRI: a tutorial overview. Neuroimage 45, S199-209. doi:10.1016/j.neuroimage.2008.11.007

Pessoa, L., 2010. Emergent processes in cognitive-emotional interactions. Dialogues Clin. Neurosci. 12, 433-448.

Poincaré, H., 2009. The foundations of science. Cornell University Library.

Posner, M.I., Boies, S.J., 1971. Components of attention. Psychol. Rev. 78, 391-408. doi:10.1037/h0031333 
Posner, M.I., Sheese, B.E., Odludaş, Y., Tang, Y., 2006. Analyzing and shaping human attentional networks. Neural Networks 19, 1422-1429.

Rabinovich, M., Huerta, R., Laurent, G., 2008. Neuroscience. Transient dynamics for neural processing. Science (80-. ). 321, 48-50. doi:10.1126/science.1155564

Rabinovich, M., Tristan, I., Varona, P., 2013. Neural Dynamics of Attentional CrossModality Control. PLoS One 8, e64406. doi:10.1371/journal.pone.0064406

Rabinovich, M., Volkovskii, A., Lecanda, P., Huerta, R., Abarbanel, H.D., Laurent, G., 2001. Dynamical encoding by networks of competing neuron groups: winnerless competition. Phys Rev Lett 87, 68102.

Rabinovich, M.I., Afraimovich, V.S., Bick, C., Varona, P., 2012a. Information flow dynamics in the brain. Phys. Life Rev. 9, 51-73. doi:http://dx.doi.org/10.1016/j.plrev.2011.11.002

Rabinovich, M.I., Afraimovich, V.S., Varona, P., 2010a. Heteroclinic Binding. Dyn. Syst. An Int. J. 25, 433-442. doi:10.1080/14689367.2010.515396

Rabinovich, M.I., Bick, C., Varona, P., 2012. The stability of information flows in the brain, in: Principles of Brain Dynamics: Global State Interactions. MIT Press, Cambridge, MA, pp. 141-164.

Rabinovich, M.I., Friston, K., Varona, P. (Eds.), 2012b. Principles of brain dynamics: global state interactions. MIT Press, Cambridge (MA).

Rabinovich, M.I., Huerta, R., Varona, P., 2006a. Heteroclinic synchronization: ultrasubharmonic locking. Phys Rev Lett 96, 141001.

Rabinovich, M.I., Huerta, R., Varona, P., Afraimovich, V.S., 2006b. Generation and reshaping of sequences in neural systems. Biol Cybern 95, 519-536. doi:10.1007/s00422-006-0121-5

Rabinovich, M.I., Huerta, R., Varona, P., Afraimovich, V.S., 2008. Transient cognitive dynamics, metastability, and decision making. PLoS Comput Biol 4, e1000072. doi:10.1371/journal.pcbi.1000072

Rabinovich, M.I., Muezzinoglu, M.K., 2010. Nonlinear dynamics of the brain: emotion and cognition. Physics-Uspekhi 53, 357-372.

doi:10.3367/UFNe.0180.201004b.0371

Rabinovich, M.I., Muezzinoglu, M.K., Strigo, I., Bystritsky, A., 2010b. Dynamical Principles of Emotion-Cognition Interaction: Mathematical Images of Mental Disorders. PLoS One 5, e12547. doi:10.1371/journal.pone.0012547

Rabinovich, M.I., Varona, P., 2011. Robust transient dynamics and brain functions. Front. Comput. Neurosci. 5, 24. doi:10.3389/fncom.2011.00024

Rabinovich, M.I., Varona, P., Selverston, A.I., Abarbanel, H.D.I., 2006c. Dynamical principles in neuroscience. Rev. Mod. Phys. 78, 1213-1265. doi:10.1103/RevModPhys.78.1213

Raichle, M.E., 2011. The Restless Brain. Brain Connect. doi:10.1089/brain.2011.0019 Raizada, R.D.S., Kriegeskorte, N., 2010. Pattern-Information fMRI: New Questions Which It Opens Up and Challenges Which Face It. Int J Imaging Syst Technol 20, 31-41.

Reeves, S.D., 2006. Creative Jazz Improvisation, Fourth. ed. Pearson.

Rissman, J., Wagner, A.D., 2012. Distributed representations in memory: insights from functional brain imaging. Annu. Rev. Psychol. 63, 101-28. doi:10.1146/annurevpsych-120710-100344 
Rouder, J.N., Morey, R.D., Cowan, N., Zwilling, C.E., Morey, C.C., Pratte, M.S., 2008. An assessment of fixed-capacity models of visual working memory. Proc Natl Acad Sci U S A 105, 5975-5979. doi:10.1073/pnas.0711295105

Sadaghiani, S., Kleinschmidt, A., 2013. Functional interactions between intrinsic brain activity and behavior. Neuroimage in press.

doi:10.1016/j.neuroimage.2013.04.100

Sänger, J., Lindenberger, U., Müller, V., 2011. Interactive brains, social minds. Commun. Integr. Biol.

Sänger, J., Müller, V., Lindenberger, U., 2012. Intra- and interbrain synchronization and network properties when playing guitar in duets. Front. Hum. Neurosci. 6, 312.

Sänger, J., Müller, V., Lindenberger, U., 2013. Directionality in hyperbrain networks discriminates between leaders and followers in guitar duets. Front. Hum. Neurosci. 7, 234.

Santosa, C.M., Strong, C.M., Nowakowska, C., Wang, P.W., Rennicke, C.M., Ketter, T.A., 2007. Enhanced creativity in bipolar disorder patients: a controlled study. J. Affect. Disord. 100, 31-39.

Sarason, I.G., Pierce, G.R., Sarason, B.R., 1996. Cognitive Interference: Theories, Methods and Findings, The LEA series in personality and clinical psychology, The LEA series in personality and clinical psychology. Lawrence Erlbaum Associates, Inc.

Schwartze, M., Rothermich, K., Schmidt-Kassow, M., Kotz, S.A., 2011. Temporal regularity effects on pre-attentive and attentive processing of deviance. Biol. Psychol. 87, 146-151.

Schwenk, C.R., 1984. Cognitive simplification processes in strategic decision-making. Strateg. Manag. J. 5, 111-128. doi:10.1002/smj.4250050203

Segall, J.M., Allen, E.A., Jung, R.E., Erhardt, E.B., Arja, S.K., Kiehl, K., Calhoun, V.D., 2012. Correspondence between structure and function in the human brain at rest. Front. Neuroinform. 6, 1-17. doi:10.3389/fninf.2012.00010

Seliger, P., Tsimring, L.S., Rabinovich, M.I., 2003. Dynamics-based sequential memory: Winnerless competition of patterns. Phys. Rev. E 67, 11905.

Singer, W., 2013. Cortical dynamics revisited. Trends Cogn. Sci. 17, 616-626. doi:10.1016/j.tics.2013.09.006

Slagter, H.A., Lutz, A., Greischar, L.L., Francis, A.D., Nieuwenhuis, S., Davis, J.M., Davidson, R.J., 2007. Mental training affects distribution of limited brain resources. PLoS Biol. 5, e138.

Stedron, J.M., Sahni, S.D., Munakata, Y., 2005. Common mechanisms for working memory and attention: the case of perseveration with visible solutions. J. Cogn. Neurosci. 17, 623-631.

Strong, C.M., Nowakowska, C., Santosa, C.M., Wang, P.W., Kraemer, H.C., Ketter, T.A., 2007. Temperament-creativity relationships in mood disorder patients, healthy controls and highly creative individuals. J. Affect. Disord. 100, 41-48.

Swallow, K.M., Zacks, J.M., Abrams, R.A., 2009. Event boundaries in perception affect memory encoding and updating. J. Exp. Psychol. Gen. 138, 236-257.

Swanson, H.L., 1999. What develops in working memory? A life span perspective. Dev Psychol 35, 986-1000. 
Tamás Kincses, Z., Johansen-Berg, H., Tomassini, V., Bosnell, R., Matthews, P.M., Beckmann, C.F., 2008. Model-free characterization of brain functional networks for motor sequence learning using fMRI. Neuroimage 39, 1950-1958.

Turner, J.H., Stets, J.E., 2005. The Sociology of Emotions. Cambridge University Press.

Van Damme, S., Legrain, V., Vogt, J., Crombez, G., 2010. Keeping pain in mind: a motivational account of attention to pain. Neurosci. Biobehav. Rev. 34, 204-213. doi:10.1016/j.neubiorev.2009.01.005

Varona, P., Rabinovich, M.I., Selverston, A.I., Arshavsky, Y.I., 2002. Winnerless competition between sensory neurons generates chaos: A possible mechanism for molluscan hunting behavior. Chaos 12, 672-677.

Venaille, A., Varona, P., Rabinovich, M.I., 2005. Synchronization and coordination of sequences in two neural ensembles. Phys Rev E Stat Nonlin Soft Matter Phys 71, 61909.

Viviani, R., Grön, G., Spitzer, M., 2005. Functional principal component analysis of fMRI data. Hum. Brain Mapp. 24, 109-29. doi:10.1002/hbm.20074

Wallas, G., 1926. The Art of Thought, The Sewanee Review. Harcourt Brace.

Wen-Kai Tsai, K., Nummenmaa, A., Witzel, T., Chang, W.T., Kuo, W.J., Lin, F.H., 2012. Multi-projection magnetic resonance inverse imaging of the human visuomotor system. Neuroimage 61, 304-313.

Wiestler, T., Diedrichsen, J., 2013. Skill learning strengthens cortical representations of motor sequences. Elife 2, e00801. doi:10.7554/eLife.00801

Woolf, C.J., 2010. What is this thing called pain? J. Clin. Invest. 120, 3742-3744.

Young, S.E., Friedman, N.P., Miyake, A., Willcutt, E.G., Corley, R.P., Haberstick, B.C., Hewitt, J.K., 2009. Behavioral disinhibition: liability for externalizing spectrum disorders and its genetic and environmental relation to response inhibition across adolescence. J. Abnorm. Psychol. 118, 117-130.

Yuste, R., MacLean, J.N., Smith, J., Lansner, A., 2005. The cortex as a central pattern generator. Nat Rev Neurosci 6, 477-483.

Zacks, J.M., Speer, N.K., Swallow, K.M., Braver, T.S., Reynolds, J.R., 2007. Event perception: a mind-brain perspective. Psychol. Bull. 133, 273-293.

Zacks, J.M., Tversky, B., 2001. Event structure in perception and conception. Psychol. Bull. 127, 3-21.

Zeeman, E., Zeeman, M., 2003. From local to global behavior in competitive LotkaVolterra systems. Trans. Am. Math. Soc. 355, 713-734.

Zeeman, M.L., 1993. Hopf bifurcations in competitive three-dimensional Lotka-Volterra systems. Dyn. Stab. Syst. 8, 189-217. 
